\title{
26. CARBONATE AND ORGANIC-CARBON CYCLES AND THE HISTORY OF UPWELLING AT DEEP SEA DRILLING PROJECT SITE 532, WALVIS RIDGE, SOUTH ATLANTIC OCEAN ${ }^{1}$
}

\author{
James V. Gardner, U.S. Geological Survey, Menlo Park, California \\ Walter E. Dean, U.S. Geological Survey, Denver, Colorado \\ and \\ Christine R. Wilson, U.S. Geological Survey, Menlo Park, California
}

\begin{abstract}
Detailed carbonate and organic-carbon stratigraphies were constructed from samples collected every $20 \mathrm{~cm}$ in a 250-m hydraulic piston core recovered at DSDP Site 532 on Walvis Ridge. This sampling interval represents about one sample every $5000 \mathrm{yr}$., based on sediment accumulation rates calculated from nannofossil biostratigraphic zones. All samples were analyzed for percent $\mathrm{CaCO}_{3}$, resulting in a detailed carbonate stratigraphy for the past $5.0 \mathrm{~m} . \mathrm{y}$. The samples for the top $110 \mathrm{~m}$ of section were also analyzed for organic carbon in order to construct a detailed organiccarbon stratigraphy for the last $2.5 \mathrm{~m} . \mathrm{y}$.

The recovered section has distinct dark-light color cycles with average periodicities of 55, 58, and 30 k.y. for the Quaternary, upper Pliocene, and lower Pliocene, respectively. Periodicities of carbonate cycles are similar to the color cycles; most carbonate minima correspond to the dark parts of color cycles. The average periodicity for carbonate cycles is about 36 k.y. Darker parts of color cycles usually contain higher concentrations of organic carbon, but the organic-carbon record does not follow the cyclicity of the color cycles in detail, at least for the last $2.5 \mathrm{~m} . \mathrm{y}$. Organic-carbon cycles have an average periodicity of about 34 k.y. for the Quaternary and upper Pliocene.

The cycles of $\mathrm{CaCO}_{3}$ and color have periodicities similar to those reported from carbonate stratigraphies from the northeast Atlantic, Caribbean, and eastern equatorial Pacific. The carbonate cycles at Site 532 are the result of external forcing, probably related to global climate, that affected fluctuations in both sediment supply from the African continental margin and productivity of siliceous organisms. The organic-carbon cycles have similar periodicities and similar changes in periodicities to those of the $\mathrm{CaCO}_{3}$ cycles.

Semiquantitative estimates of diatom abundance from smear slides and concentrations of biogenic $\mathrm{SiO}_{2}$ calculated from chemical analyses suggest that upwelling at Site 532 was minor until about $3 \mathrm{~m}$.y. ago. The Benguela-Current upwelling system either began at that time or, more likely, migrated into the area of Site 532, where it prevailed until some time between about 1.2 and 0.5 m.y. ago. The increase and decline of upwelling in the area of Site 532, however, did not disturb the trend of cyclicities of carbonate and organic carbon. The latest change in conditions at Site 532 was an increase in intensity of bottom currents during the past $0.5 \mathrm{~m}$.y. that winnowed nannofossils, diatoms, and clay and left a lag deposit represented by a foraminifer-rich facies.
\end{abstract}

\section{INTRODUCTION}

Walvis Ridge is a structural spur that projects southwestward from the continental margin of South Africa off Namibia and is beneath the cold, nutrient-rich, Benguela-Current upwelling system (Fig. 1). DSDP Site 532 is located on the eastern part of Walvis Ridge at a water depth of $1331 \mathrm{~m}$ in a trough with a relatively thick sediment section. The site is about $2 \mathrm{~km}$ from DSDP Site 362 (Bolli, Ryan, et al., 1978) which was rotary drilled, but unfortunately the upper $200 \mathrm{~m}$ of the section is badly disturbed. The data from Site 362 , however, suggest that increases in abundances of diatoms and organic carbon may be linked to the onset and development of the Benguela-Current upwelling system (Bolli, Ryan, et al., 1978; Diester-Haass and Schrader, 1979; Siesser, 1980). The Benguela-Current upwelling system apparently began about $10 \mathrm{~m} . y$. ago in the early late-Miocene, with progressively more intense upwelling in the Pliocene and Pleistocene. Seisser (1980) concluded that increases in diatoms and organic carbon reflect the estab-

\footnotetext{
${ }^{1}$ Hay, W. W., Sibuet, J.-C., et al., Init. Repts. DSDP, 75: Washington (U.S. Govt. Printing Office).
}

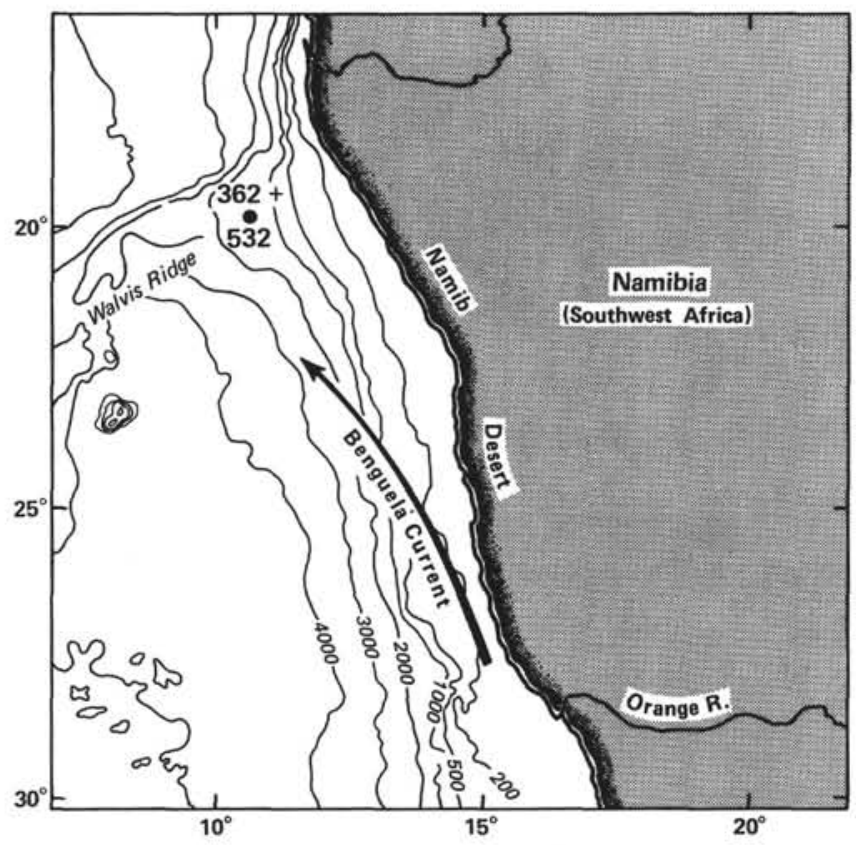

Figure 1. Map showing general bathymetry of the continental margin off southwest Africa and locations of DSDP Sites 362 and 532. 
lishment of the strong southeast trade winds that drive the upwelling system today.

Hydraulic piston coring at Site 532 recovered a nearly continuous section of late Neogene and Quaternary age. Biostratigraphic data from Site 532 indicate that all nannofossil zones of Ellis (1981) are present (Site 532 summary chapter, this volume). The section from Site 532 was divided into three lithostratigraphic subunits based on relative proportions of siliceous and calcareous microfossils and nonbiogenic material (clay) (Fig. 2; see also Site 532 summary chapter, this volume). The amount of clay steadily decreases from the bottom to the top of the section. The section from $290 \mathrm{~m}$ (bottom of Hole 532B) to $114 \mathrm{~m}$ sub-bottom consists of nannofossil marl with rare $(<5 \%)$ planktonic foraminifers and rare $(<1 \%)$ diatoms. The section from 114 to $50 \mathrm{~m}$ sub-bottom consists of nannofossil diatom marl that contains about equal amounts of diatoms, nannofossils, and clay (Fig. 2). The abundance of diatoms decreases and the abundance of nannofossils increases above $50 \mathrm{~m}$ sub-bottom. Dia- toms are rare above $20 \mathrm{~m}$ sub-bottom, and foraminifers increase in abundance and make up more than $50 \%$ of the sediment at the top of the section (Fig. 2).

Preliminary shipboard descriptions of the section at Site 532 noted cycles of dark- and light-colored sediment that contain abrupt changes in abundances of foraminifers and diatoms (Site 532 chapter, this volume), but these changes in microfossil abundance are not systematic within the color cycles. The concentration of $\mathrm{CaCO}_{3}$, however, does change systematically within individual color cycles and is highest in the lightest colored part of a cycle. The changes in color and abundances of siliceous and calcareous microfossils may reflect changes in productivity associated with the intensity of the Benguela Current upwelling system. We therefore chose this site to construct detailed carbonate and organic-carbon stratigraphies to detect any cyclic variations in $\mathrm{CaCO}_{3}$ and organic carbon that can be correlated with cyclic variations in other sediment parameters.

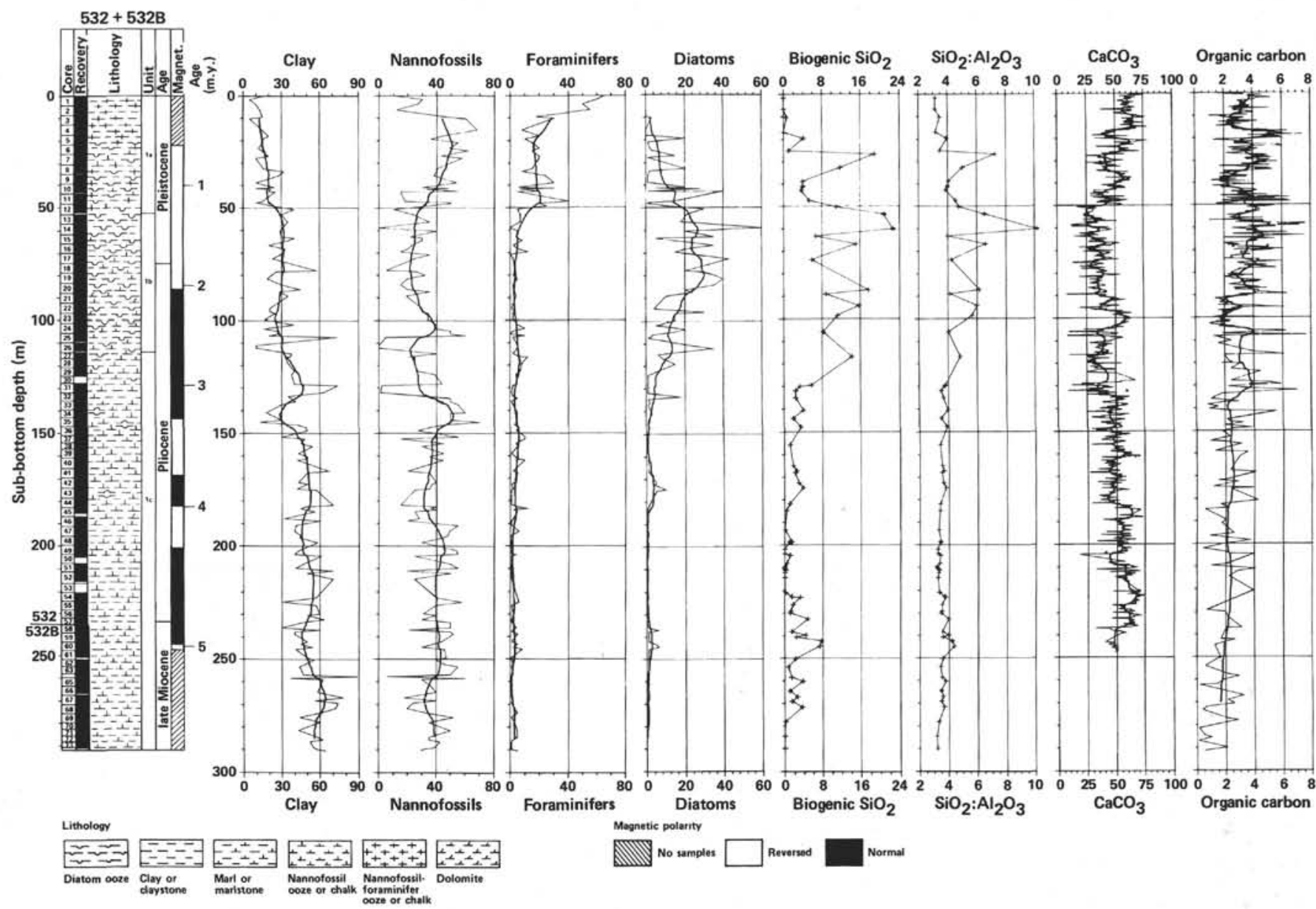

Figure 2. Lithology, age, magnetics, percentages of nonbiogenic material (clay), nannofossils, foraminifers, diatoms, biogenic silica, $\mathrm{CaCO}_{3}$, and organic carbon, and $\mathrm{SiO}_{2}: \mathrm{Al}_{2} \mathrm{O}_{3}$ ratio, at DSDP Site 532. Percentages of clay, nannofossils, foraminifers, and diatoms are from smear-slide estimates (Site 532 summary chapter, this volume). Percent biogenic silica and the $\mathrm{SiO}_{2}: \mathrm{Al}_{2} \mathrm{O}_{3}$ ratio are from Dean and Parduhn (this volume). Percentages of $\mathrm{CaCO}_{3}$ and organic carbon are from Table 1. Smoothed curves through the smear-slide data were computed using a 5-point weighted moving average. Smoothed curves through the $\mathrm{CaCO}_{3}$ and organic-carbon data were computed using a 15-point weighted moving average. 


\section{DESCRIPTION OF THE CYCLES}

\section{Color Cycles}

The most noticeable characteristic of the sediment at Site 532 is the cyclic dark and light variations in sediment color (Fig. 3). The upper $160 \mathrm{~m}$ of the section has dark-light alternations mostly of yellow brown (hue $5 \mathrm{Y}$ ); the section below $160 \mathrm{~m}$ has cycles of light and dark chromas mostly of greenish olive (hue 10Y). The contacts between light and dark interbeds usually are gradational over about 10 to $20 \mathrm{~cm}$. The overall color of the section is lightest in the lower Pliocene, but becomes darker in the middle Pliocene. The intensity and frequency of darker colored layers are highest in the early Pliocene. The overall color again becomes lighter in the uppermost Quaternary. We determined the periodicities of the color cycles by counting the number of cycles between time datums established by nannofossil zones.

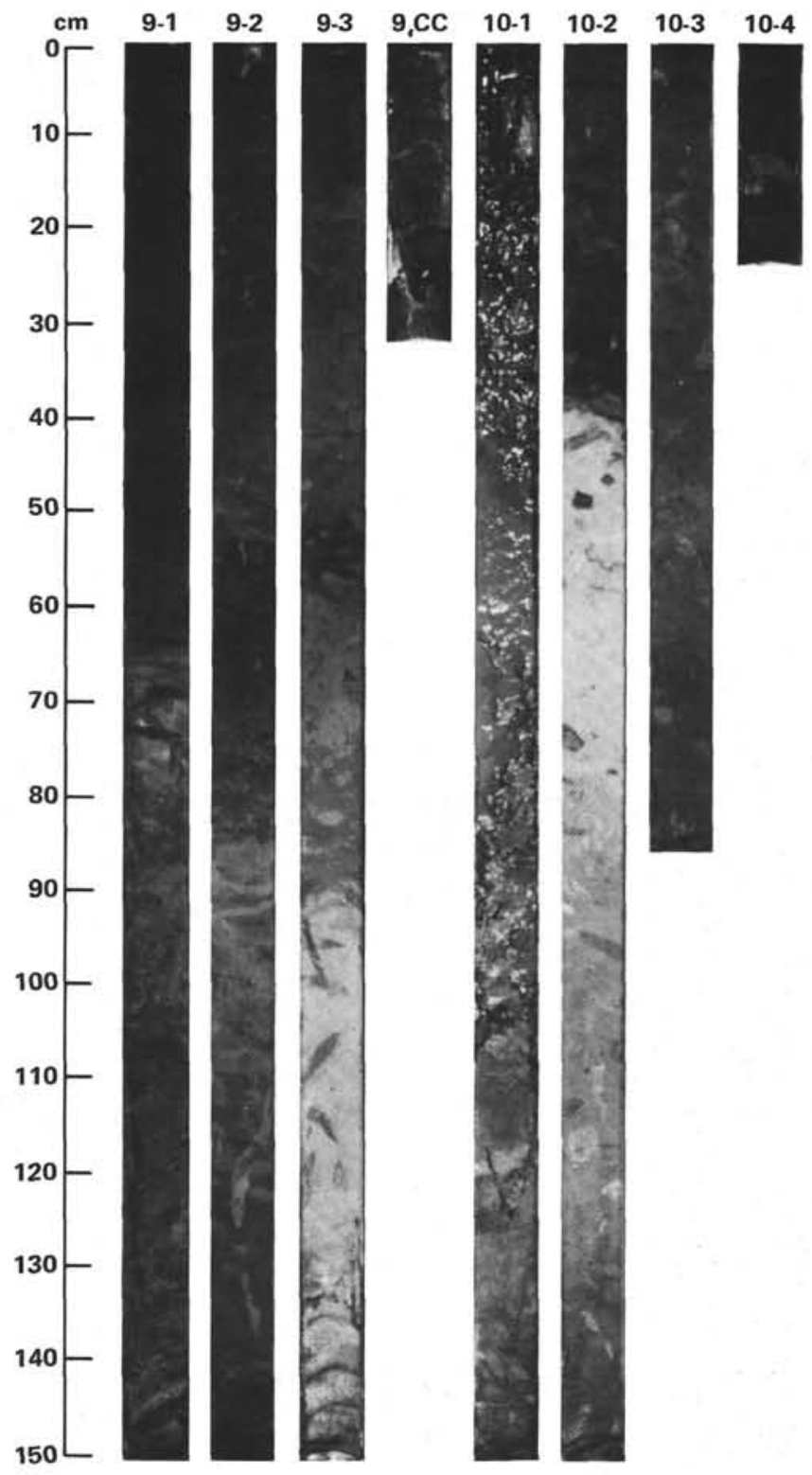

Figure 3. Photograph of Core 10, Hole 532.
The cycles have periodicities of 55,58 , and $30 \mathrm{k} . \mathrm{y}$. for the Quaternary, upper Pliocene, and lower Pliocene sections, respectively.

\section{Carbonate and Organic-Carbon Cycles}

The entire section in Hole 532 was sampled at $20 \mathrm{~cm}$ intervals for analyses of $\mathrm{CaCO}_{3}$. This sampling resulted in 1057 samples with an average sampling interval of about $5000 \mathrm{yr} . / \mathrm{sample}$. All samples were analyzed for $\mathrm{CaCO}_{3}$ using the gasometric technique of Hulsemann (1966). One-hundred fifty-three shipboard analyses of $\mathrm{CaCO}_{3}$ by the carbonate-bomb method (Muller and Gastner, 1971) from Holes 532 and 532B were merged with the larger gasometric data set (Table 1). Fourhundred and twenty of the $20-\mathrm{cm}$ carbonate samples between 0 and $109 \mathrm{~m}$ sub-bottom were also analyzed for organic carbon by the LECO method (Table 2). An additional 50 analyses of organic carbon by Meyers, Brassell, and Huc, et al. (this volume) from the same stratigraphic interval were merged with the LECO data. The only analyses of organic carbon that we have for the interval between 109 and $250 \mathrm{~m}$ sub-bottom are from Meyers, Brassell, and Huc (this volume).

The results of analyses of $\mathrm{CaCO}_{3}$ and organic carbon, listed in Tables 1 and 2 and plotted in Figure 2, show that the average concentration of $\mathrm{CaCO}_{3}$ tends to decrease from about $60 \%$ at the bottom of the section to about $25 \%$ at $55 \mathrm{~m}$, and then to increase to almost $70 \%$ at the top of the section. Stratigraphic variations in organic-carbon concentration are roughly opposite to those of $\mathrm{CaCO}_{3}$. Organic carbon increases from about $2 \%$ at the bottom of the section, to a maximum of almost $4 \%$ at about $55 \mathrm{~m}$ sub-bottom. The most striking feature of the records, however, is the high-frequency fluctuations of both $\mathrm{CaCO}_{3}$ and organic carbon.

Calcium carbonate cycles were defined as a section of the record between successive $\mathrm{CaCO}_{3}$ minima. These cycles correspond to the $C$ cycles of Gardner (1982). We define an organic-carbon cycle as the interval between successive maxima on the organic-carbon profile from 0 to $109 \mathrm{~m}$ sub-bottom (0 to 2.4 m.y.) (Fig. 2).

We constructed profiles of $\mathrm{CaCO}_{3}$ and organic carbon versus time using the ages assigned to nannofossil zones (Ellis, 1981; Site 532 summary chapter, this volume). However, even though all late Neogene and Quaternary nannofossil zones were identified (Steinmetz and Stradner, this volume), the nannofossil zonation used is based on western equatorial Pacific floras (Ellis, 1981), and they may not be strictly applicable to the eastern South Atlantic. We encountered problems when we plotted age as determined by the nannofossil zonation versus depth for Site 532. An apparent expanded section with a sedimentation rate of $106 \mathrm{~m} / \mathrm{m}$.y. was immediately preceded by an apparent abbreviated section with a sedimentation rate of $5 \mathrm{~m} / \mathrm{m}$.y. (Fig. 4). When $\mathrm{CaCO}_{3}$ is plotted versus age using this time scale, artificial expansions and compressions of parts of the section become apparent (Fig. 5A). We modified the zonation by using a correlation with the zonation of Martini (1971) (Fig. 6). The plot of age versus depth using the Martini zonation (Fig. 4) shows that the expanded and compressed sections were not eliminated, but only 
Table 1. Calcium carbonate data, Site 532.

\begin{tabular}{|c|c|c|c|c|c|c|c|c|c|c|c|c|c|}
\hline Depth-m & $2 \mathrm{CaCO}$ & Depth-m & z. $\mathrm{CaCO} 3$ & Depth-m & z $\mathrm{CaCO}$ & Depth-m & $2 \mathrm{CaCO3}$ & Depth-m & $2 \mathrm{CaCO} 3$ & Depth-m & x $\mathrm{CaCO}$ & Depth-a & z $\mathrm{CaCO} 3$ \\
\hline .20 & 75.2 & 9.40 & 64.6 & 18.00 & 44.2 & 26.60 & 48.6 & 36.00 & 58.3 & 44.60 & 28.6 & 53.20 & 26.3 \\
\hline .40 & 62.5 & 9.60 & 69.4 & 18.16 & $41.0 \bullet$ & 26.75 & $40.0 \bullet$ & 36.20 & 54.5 & 44.80 & 39.2 & 53.40 & 26.2 \\
\hline .60 & 73.6 & 9.80 & 66.0 & 18.20 & 39.4 & 26.80 & 38.7 & 36.50 & 53.7 & 45.00 & 36.2 & 53.60 & 24.7 \\
\hline .80 & 67.9 & 10.10 & 71.2 & 18.40 & 36.7 & 27.00 & 58.3 & 36.70 & 56.0 & 45.30 & 36.1 & 53.80 & 22.7 \\
\hline 1.00 & 54.6 & 10.19 & $73.0 \bullet$ & 18.60 & 30.8 & 27.20 & 46.7 & 36.90 & 55.4 & 45.50 & 28.8 & 54.10 & 26.6 \\
\hline 1.20 & 61.3 & 10.30 & 76.8 & 18.90 & 61.6 & 27.40 & 41.3 & 37.10 & 57.4 & 45.70 & 41.8 & 54.30 & 23.5 \\
\hline 1.40 & 59.2 & 10.50 & 69.9 & 19.10 & 41.4 & 27.70 & 25.9 & 37.30 & 65.1 & 45.79 & $53.0 \bullet$ & 54.43 & 27.0 \\
\hline 1.57 & $68.0 \bullet$ & 10.70 & 74.9 & 19.30 & 46.9 & 27.90 & 36.2 & 37.38 & $42.0 \bullet$ & 45.90 & 58.5 & 54.50 & 32.5 \\
\hline 1.70 & 64.9 & 10.90 & 63.8 & 19.50 & 42.3 & 28.10 & 37.1 & 37.50 & 63.8 & 46.10 & 48.4 & 54.70 & 23.2 \\
\hline 1.90 & 64.2 & 11.10 & 56.1 & 19.70 & 44.7 & 28.30 & 39.6 & 37.70 & 61.5 & 46.30 & 31.0 & 55.60 & 38.5 \\
\hline 2.10 & 58.1 & 11.30 & 64.9 & 19.90 & 42.5 & 28.50 & 35.1 & 38.00 & 61.6 & 46.50 & 36.5 & 55.80 & 31.9 \\
\hline 2.30 & 64.5 & 11.60 & 61.8 & 20.10 & 32.6 & 28.70 & 44.0 & 38.20 & 63.3 & 46.80 & 45.4 & 56.00 & 22.2 \\
\hline 3.20 & 52.2 & 11.80 & 61.6 & 20.40 & 57.3 & 28.90 & 40.2 & 38.40 & 54.1 & 47.01 & 54.0 & 56.20 & 35.9 \\
\hline 3.40 & 56.3 & 12.00 & 60.8 & 20.60 & 63.3 & 29.20 & 46.6 & 38.60 & 59.7 & 47.13 & $41.0 \bullet$ & 56.29 & 41.0 \\
\hline 3.60 & 63.6 & 12.20 & 66.8 & 20.80 & 72.6 & 29.40 & 45.2 & 38.80 & 63.0 & 47.20 & 51.8 & 56.40 & 34.0 \\
\hline 3.72 & $67.0 \bullet$ & 12.40 & 65.5 & 21.00 & 78.9 & 29.60 & 57.5 & 39.00 & 58.0 & 47.40 & 61.6 & 56.60 & 40.6 \\
\hline 3.80 & 61.4 & 12.46 & $58.0 \bullet$ & 21.16 & $70.0 \bullet$ & 29.80 & 54.2 & 39.03 & $58.0 \bullet$ & 47.60 & 57.2 & 56.77 & 51.9 \\
\hline 4.00 & 60.5 & 12.60 & 58.7 & 21.20 & 73.6 & 30.60 & 37.5 & 39.20 & 49.1 & 47.80 & 37.9 & 57.00 & 23.0 \\
\hline 4.20 & 58.2 & 12.80 & 70.6 & 21.40 & 69.5 & 30.60 & 41.6 & 39.40 & 50.7 & 48.00 & 37.6 & 57.20 & 34.8 \\
\hline 4.20 & 62.3 & 13.00 & 74.5 & 21.60 & 68.6 & 30.75 & 24.8 & 39.46 & 50.4 & 48.09 & $55.0 \bullet$ & 57.40 & 25.1 \\
\hline 4.40 & 62.6 & 13.04 & $61.9 \bullet$ & 21.80 & 50.2 & 30.80 & 46.4 & 39.60 & 56.3 & 48.20 & 35.9 & 57.60 & 34.5 \\
\hline 4.60 & 59.8 & 13.20 & 58.7 & 22.00 & 55.3 & 31.00 & 41. & 39.80 & 54.3 & 48. & & 57.80 & \\
\hline 4.80 & 58.3 & 13.40 & 66.6 & 22.20 & 64.0 & 31.20 & 32.5 & 40.00 & 51.7 & 0 & 40 & 58.00 & 26.3 \\
\hline 5.00 & 54.9 & 13.60 & 64.5 & 22.40 & 62.1 & 31.40 & 35.0 & 40.20 & 50.2 & 48.44 & 34.7 & 58.20 & 15.3 \\
\hline 5.20 & 57.7 & 13.80 & 65.5 & 22.60 & 58.5 & 31.60 & 36.6 & 40.40 & 48.5 & 48.60 & 42.3 & 58.51 & 11.6 \\
\hline 5.40 & 61.5 & 14.00 & 55.3 & 22.80 & 67.0 & 31.80 & 47.5 & 40.60 & 42.1 & 48.79 & 42.0 & 58.68 & 15.0 \\
\hline 5.70 & 62.8 & 14.20 & 64.9 & 23.00 & 57.0 & 32.10 & S1. & 40.90 & 38.8 & 48.80 & 41.3 & 58.70 & 19.4 \\
\hline 5.90 & 65.9 & 14.47 & $68.0 \bullet$ & 23.30 & 54.2 & 32.30 & 43.4 & 41.10 & 53.6 & 49.00 & 45.6 & 58.90 & 25.3 \\
\hline 6.10 & 67.9 & 14.50 & 67.6 & 23.50 & 52.1 & 32.50 & 55.2 & 41.30 & 57.6 & 49.20 & 55 & 59.10 & 12.8 \\
\hline 6.30 & 58.1 & 14.70 & 78.8 & 23.65 & $34.0 \bullet$ & 32.60 & $39.0 \bullet$ & 41.34 & $58.0 \bullet$ & 49.40 & 56.0 & 59.30 & 38.6 \\
\hline 6.50 & 64.7 & 14.90 & 69.3 & 23.70 & 43.1 & & 40 & 41.50 & 43. & 49.60 & 53. & 59.50 & 44.3 \\
\hline & 52 & & & 23. & & 32. & & 41. & 49 & & 53 & 59.70 & 31.2 \\
\hline 6.90 & 44.0 & 15.30 & 64.1 & 24.10 & 55.7 & 33.10 & 43. & 41. & $50.0 \bullet$ & & 32 & 60.10 & 43.4 \\
\hline 7.06 & $38.0 \bullet$ & 15.30 & 68.1 & 24.30 & 57.0 & 33.30 & 41.8 & 41.90 & 57. & 50.19 & 33.0 & 60.28 & $44.0 \bullet$ \\
\hline 7.20 & 57.2 & 15.50 & 66.7 & 24.50 & 59.6 & 33.60 & 40.6 & 42.10 & 59.6 & 50.21 & 34.3 & 60.30 & 46.4 \\
\hline 7.40 & 62. & 15.70 & 66.5 & 24.54 & $62.0 \bullet$ & 33.80 & 41.6 & 42.40 & 50. & 50.40 & 20.1 & 60. & 47.3 \\
\hline & 64.2 & 16.00 & 61.6 & 24.8 & 56.2 & 34. & 43 & 42. & 48 & & 16 & & 44.8 \\
\hline 7.60 & 62.0 & 16.20 & 63.4 & 25.0 & 53. & 34.20 & 34.2 & 42. & 50. & 50.80 & 22.3 & 60.90 & 39.4 \\
\hline 7.80 & 62.1 & 16.40 & 56.1 & 25.20 & 56.1 & 34.40 & 36.3 & 42.80 & 51.3 & 51.10 & 33.9 & 61.40 & 36.0 \\
\hline 8.00 & 56.1 & 16.60 & 42.2 & 25.40 & 60.4 & 34.96 & $62.0 \bullet$ & 43.00 & 44.9 & 51.30 & 27.8 & 61.60 & 27.8 \\
\hline 8.20 & 5 & 17.00 & 42 & 25. & 71 & 35.00 & 43 & 43. & 32. & 51. & 31 & 61.80 & 26.8 \\
\hline & & & & 25 & 68. & 35. & 54 & 43. & 31 & 52. & 30 & 62. & 31.6 \\
\hline 8. & 51 & 17.40 & 49.2 & 26.00 & 55.0 & 35.40 & 50.3 & 44. & 39. & 52.80 & 28.5 & 62.20 & 19.4 \\
\hline 9.00 & 56.1 & 17.60 & 39.4 & 26.20 & 45.5 & 35.60 & 53.2 & 44.20 & 44 & 53.00 & 27.9 & 62.40 & 23.1 \\
\hline 9.20 & 56.3 & 17.80 & 39.1 & 26.40 & 46.3 & 35.80 & 59.4 & 44.40 & 34.6 & 53.09 & 25.0 & 62.60 & 30.3 \\
\hline
\end{tabular}

moved from the lower Pliocene to the upper Pliocene. Because all of the nannofossil zones and sub-zones of Ellis (1981) were identified, and because we found no sedimentological evidence for a greatly expanded section, nor any indication of an unconformity, we developed a preferred zonation for Site 532, based mainly on the Martini (1971) time-scale (Fig. 6).

Successive age datums from our preferred zonation were used to calculate an average sedimentation rate between datums. Each sample was then assigned an age by linear interpolation. The resulting plots of percent $\mathrm{CaCO}_{3}$ and organic carbon versus age are shown in Figures $5 \mathrm{~B}$ and $5 \mathrm{C}$. We subdivided the age axes of the $\mathrm{CaCO}_{3}$ and organic carbon versus age plots into 0.5 m.y. intervals and counted the number of cycles in each $0.5 \mathrm{~m}$.y. segment. We calculated the average percentages of $\mathrm{CaCO}_{3}$, organic carbon, and biogenic silica for each 0.5 m.y. interval (Table 3). We also calculated the periodicities of $\mathrm{CaCO}_{3}$, organic carbon, and dark beds (Table 4).

The periodicities of the $\mathrm{CaCO}_{3}$ cycles (Table 4) range from a low of $26 \mathrm{k}$.y. to a maximum of $49 \mathrm{k} . \mathrm{y}$. and aver- age about $36 \mathrm{k} . \mathrm{y}$. Most of the $\mathrm{CaCO}_{3}$ minima correspond to the dark parts of color cycles (Figs. 3 and 5B), and most of the maxima correspond to the lightest parts of color cycles.

The periodicities of the organic-carbon cycles (Table 4) range from 28 k.y. to about 36 k.y., with an average of about $34 \mathrm{k} . \mathrm{y}$. for the interval 0 to $2.5 \mathrm{my}$. Although all dark-colored beds correspond to an organic-carbon maximum, the relationship between organic-carbon concentration and dark and light beds is not so clear as for $\mathrm{CaCO}_{3}$. Many of the organic-carbon maxima do not appear to correspond to a dark bed. As a result, the organic-carbon cycles have a higher frequency (shorter period) than those of the dark beds (Table 4).

The $\mathrm{CaCO}_{3}$ and organic-carbon profiles (Fig. 2) also show lower frequency cycles on a scale of 5 to $10 \mathrm{~m}$ thick, which corresponds to an average periodicity of about 150 k.y. These longer cycles are best seen in the smoothed curves for parts of the profiles between 40 and $80 \mathrm{~m}$ sub-bottom (Figs. 2 and 10 ).

A color cycle just over $2 \mathrm{~m}$ long from Core 10 , Section 2, to Core 11, Section 1 (mid-Pleistocene) was 
Table 1. (Continued.)

\begin{tabular}{|c|c|c|c|c|c|c|c|c|c|c|c|c|c|}
\hline Depth-n & ₹ $\mathrm{CaCO} 3$ & Depth-m & z $\mathrm{CaCO} 3$ & Depth-m & z $\mathrm{CaCO}^{3}$ & Depth-n & $\mathrm{z} \mathrm{CaCO}$ & Depth-m & $2 \mathrm{CaCu} 3$ & Depth-m & $2 \mathrm{CaCO} 3$ & Depth-n & z $\mathrm{CaCO}_{3}$ \\
\hline 62.88 & 35.5 & 70.81 & $43.0 \bullet$ & 79.40 & 38.9 & 88.80 & 32.1 & 96.80 & 56.6 & 105.98 & 24.0 & 116.36 & $34.0 \bullet$ \\
\hline 63.06 & $27.0 \bullet$ & 71.00 & 45.7 & 79.60 & 34.0 & 89.00 & 33.1 & 96.99 & $45.0 \bullet$ & 106.20 & 41.7 & 116.50 & 35.1 \\
\hline 63.10 & 28.2 & 71.20 & 43.7 & 79.80 & 37.3 & 89.30 & 16.4 & 97.02 & 46.3 & 106.40 & 52.3 & 116.70 & 31.5 \\
\hline 63.30 & 20.9 & 71.40 & 41.1 & 80.00 & 40.3 & 89.39 & $8.0 \bullet$ & 97.20 & 51.9 & 106.60 & 47.0 & 116.91 & 25.4 \\
\hline 63.50 & 14.4 & 71.70 & 34.0 & 80.20 & 39.8 & 89.50 & 41.3 & 97.40 & 52.6 & 106.90 & 51.2 & 117.20 & 31.8 \\
\hline 63.70 & 20.9 & 71.90 & 35.5 & 80.50 & 45.9 & 89.70 & 42.1 & 97.60 & 59.4 & 107.10 & 42.8 & 117.40 & 26.8 \\
\hline 63.90 & 31.4 & 72.10 & 38.3 & 80.70 & 54.6 & 89.90 & 38.0 & 97.80 & 63.8 & 107.21 & $31.0 \bullet$ & 117.60 & 44.9 \\
\hline 64.10 & 30.1 & 72.30 & 36.6 & 80.90 & 49.2 & 90.10 & 36.3 & 98.10 & 52.0 & 107.30 & 25.3 & 117.80 & 33.1 \\
\hline 64.39 & 40.4 & 72.31 & $32.0 \bullet$ & 81.12 & $39.0 \bullet$ & 90.30 & 38.1 & 98.30 & 51.0 & 107.50 & 8.7 & 117.92 & 36.9 \\
\hline 64.54 & 42.0 & 72.50 & 36.8 & 81.30 & 29.4 & 90.50 & 36.8 & 98.50 & 47.8 & 107.70 & 23.0 & 118.40 & 28.2 \\
\hline 64.61 & 42.5 & 72.70 & 38.3 & 81.49 & 19.1 & 90.80 & 49.8 & 98.70 & 53.8 & 107.90 & 47.6 & 118.60 & 32.0 \\
\hline 64.80 & 34.7 & 72.90 & 39.6 & 81.68 & 26.2 & 91.00 & 49.0 & 98.90 & 54.0 & 108.10 & 54.7 & 118.78 & 25.5 \\
\hline 65.00 & 28.7 & 73.20 & 47.2 & 81.80 & 39.3 & 91.20 & 49.7 & 99.10 & 59.4 & 108.30 & 46.1 & 119.08 & $27.0 \bullet$ \\
\hline 65.20 & 35.6 & 73.40 & 46.2 & 82.00 & 39.1 & 91.40 & 53.2 & 99.30 & 58.2 & 108.43 & 56.5 & 119.12 & 29.5 \\
\hline 65.40 & 39.4 & 73.60 & 47.3 & 82.20 & 38.7 & 91.48 & $48.0 \bullet$ & 99.60 & 62.0 & 109.80 & 46.0 & 119.30 & 33.2 \\
\hline 65.60 & 38.2 & 73.80 & 52.8 & 82.40 & 37.7 & 91.60 & 52.2 & 99.74 & $58.0 \bullet$ & 110.00 & 46.5 & 119.50 & 29.0 \\
\hline 65.75 & 39.8 & 73.81 & $47.0 \bullet$ & 82.60 & 32.8 & 91.80 & 58.7 & 99.80 & 61.8 & 110.09 & $40.0 \bullet$ & 119.70 & 26.4 \\
\hline 65.80 & 27.2 & 73.91 & $32.0 \bullet$ & 82.69 & $28.0 \bullet$ & 92.00 & 53.0 & 100.00 & 62.2 & 110.20 & 41.5 & 119.90 & 34.6 \\
\hline 65.90 & 39.1 & 74.00 & 43.6 & 82.80 & 34.5 & 92.20 & 43.1 & 100.20 & 63.0 & 110.40 & 34.2 & 120.00 & 55.1 \\
\hline 66.00 & 27.7 & 74.20 & 37.2 & 83.00 & 34.3 & 92.30 & 50.8 & 101.00 & 57.6 & 110.60 & 31.0 & 120.10 & 38.2 \\
\hline 66.20 & 43.2 & 74.60 & 30.3 & 83.40 & 38.8 & 92.40 & 46.1 & 101.20 & 56.2 & 110.80 & 28.1 & 120.29 & 29.9 \\
\hline 66.39 & 52.3 & 74.80 & 28.1 & 83.60 & 35.5 & 92.60 & 42.5 & 101.40 & 55.7 & 111.00 & 39.7 & 120.56 & 32.2 \\
\hline 66.60 & 53.7 & 75.00 & 14.0 & 83.80 & 33.0 & 92.80 & 43.7 & 101.60 & 61.4 & 111.15 & 45.0 & 120.80 & 36.9 \\
\hline 66.78 & $48.0 \bullet$ & 75.20 & 13.5 & 83.90 & $31.0 \bullet$ & 93.00 & 41.6 & 101.80 & 57.5 & 111.30 & 47.3 & 121.00 & 36.7 \\
\hline 66.80 & 49.4 & 75.31 & $21.0 \bullet$ & 84.00 & 35.8 & 93.20 & 43.2 & 101.89 & $55.0 \bullet$ & 111.50 & 42.2 & 121.20 & 36.6 \\
\hline 67.00 & 50.0 & 75.40 & 30.1 & 84.21 & 28.3 & 93.36 & $40.0 \bullet$ & 102.00 & 58.3 & 111.70 & 34.9 & 121.40 & 28.3 \\
\hline 67.30 & 46.1 & 75.60 & 38.9 & 84.40 & 29.9 & 93.40 & 40.8 & 102.20 & 56.7 & 111.90 & 42.6 & 121.60 & 34.3 \\
\hline 67.50 & 34.4 & 75.80 & 40.1 & 84.60 & 35.4 & 93.70 & 37.9 & 102.50 & 52.6 & 112.10 & 41.9 & 121.80 & 34.5 \\
\hline 67.70 & 46.7 & 76.10 & 32.1 & 84.90 & 36.3 & 93.90 & 35.9 & 102.70 & 54.6 & 112.30 & 40.7 & 122.00 & 33.1 \\
\hline 67.90 & 35.0 & 76.30 & 34.3 & 85.10 & 40.1 & 94.10 & 39.4 & 102.90 & 51.4 & 112.50 & 35.9 & 122.20 & 31.4 \\
\hline 68.10 & 28.6 & 76.50 & 26.3 & 85.30 & 40.7 & 94.30 & 46.4 & 103.10 & 48.7 & 112.80 & 32.6 & 122.40 & $45.0 \bullet$ \\
\hline 68.30 & 23.4 & 76.70 & 27.5 & 85.51 & 41.5 & 94.50 & 53.3 & 103.30 & 49.3 & 113.00 & 33.3 & 122.50 & 47.1 \\
\hline 68.32 & $19.0 \bullet$ & 76.81 & $20.0 \bullet$ & 86.40 & 26.5 & 94.70 & 45.9 & 103.50 & 48.7 & 114.20 & 43.4 & 122.70 & 50.1 \\
\hline 68.50 & 38.1 & 76.90 & 26.4 & 86.60 & 28.6 & 94.86 & $41.0 \bullet$ & 103.57 & $50.0 \bullet$ & 114.40 & 44.1 & 122.90 & $48.1 \bullet$ \\
\hline 68.80 & 28.4 & 77.10 & 28.3 & 86.80 & 36.9 & 94.90 & 44.3 & 103.70 & 56.9 & 114.60 & 42.6 & 123.10 & 35.0 \\
\hline 69.00 & $25.0 \bullet$ & 77.30 & 37.8 & 86.91 & 31.0 & 95.20 & 31.3 & 104.00 & 50.7 & 114.80 & 42.7 & 123.90 & 25.0 \\
\hline 69.01 & 29.8 & 77.60 & 41.6 & 87.00 & 36.7 & 95.40 & 19.4 & 104.20 & 50.7 & 114.86 & $32.0 \bullet$ & 124.05 & 27.7 \\
\hline 69.20 & 33.5 & 77.80 & 20.6 & 87.20 & 38.5 & 95.60 & 28.4 & 104.40 & 48.5 & 115.00 & 38.9 & 124.23 & 38.9 \\
\hline 69.40 & 27.2 & 78.00 & 29.9 & 87.41 & 36.9 & 95.97 & 29.4 & 104.60 & 44.7 & 115.20 & 43.7 & 124.43 & 50.7 \\
\hline 69.60 & 31.3 & 78.20 & 34.8 & 87.60 & 40.0 & 96.00 & 35.8 & 104.73 & $23.0 \bullet$ & 115.41 & 38.6 & 127.60 & 67.4 \\
\hline 69.80 & 48.2 & 78.40 & 38.7 & 87.80 & 42.6 & 96.20 & 34.3 & 104.80 & 38.9 & 115.70 & 47.4 & 128.00 & 54.9 \\
\hline 70.00 & 28.4 & 78.60 & 40.7 & 88.00 & 40.1 & 96.30 & $32.0 \bullet$ & 105.40 & 39.8 & 115.90 & 37.8 & 128.20 & 55.2 \\
\hline 70.20 & 22.8 & 78.80 & 39.5 & 88.20 & 41.7 & 96.40 & 41.5 & 105.60 & 26.0 & 116.10 & 10.1 & 128.41 & 52.2 \\
\hline 70.40 & 33.8 & 79.00 & 43.5 & 88.40 & 41.5 & 96.40 & 41.5 & 105.71 & $11.0 \bullet$ & 116.16 & $13.0 \bullet$ & 128.60 & 42.3 \\
\hline 70.60 & 45.8 & 79.18 & 42.5 & 88.60 & 42.3 & 96.60 & 43.6 & 105.80 & 10.2 & 116.30 & 40.6 & 128.80 & 39.4 \\
\hline
\end{tabular}

studied in detail. Figure 7 is a composite of data for this interval (Site 532, Meyers, Brassell, and Huc, this volume and this chapter).

The color changes from moderately light values at 41.5 and $44 \mathrm{~m}$ sub-bottom to very dark values at 41.3 and $43.5 \mathrm{~m}$ sub-bottom (Fig. 7). The lower dark section at $43.5 \mathrm{~m}$ has moderate concentrations of $\mathrm{CaCO}_{3}$ and low Rock-Eval $\mathrm{S}_{2}$ values (see discussion below for explanation of Rock-Eval). The section from $43.5 \mathrm{~m}$ to $41.5 \mathrm{~m}$ sub-bottom shows a steady trend to lighter colors and an increase in $\mathrm{CaCO}_{3}$ from a low of $23 \%$ at $43.4 \mathrm{~m}$ to more than $50 \%$ between $42.9 \mathrm{~m}$ and the top of the cycle. For some reason there is no decrease in percent $\mathrm{CaCO}_{3}$ that corresponds to the sharp upper color change that marks the boundary with the overlying cycle. In most other cycles, a marked color change is accompanied by a sharp change in percent $\mathrm{CaCO}_{3}$.

The concentration of organic carbon decreases from over $4 \%$ in the dark beds to about $1 \%$ at $41.3 \mathrm{~m}$. For this cycle, there is a good correspondence between color and organic-carbon concentration. As mentioned above, however, not all organic-carbon maxima correspond to dark beds. Figure 7 shows that, in general, there is a good correspondence between $\mathrm{CaCO}_{3}$ and organic car- bon, which recapitulates the general inverse relationship between these two variables over the entire section at Site 532 (see Figs. 10 and 11).

The Rock-Eval $\mathrm{S}_{2}$ values (in mg of hydrocarbons per $\mathrm{g}$ of sediment) is a rough estimate of the amount of lipid-rich marine organic matter. When normalized to the amount of organic carbon in the sediment, the $S_{2}$ values give the hydrogen index in $\mathrm{mg}$ hydrocarbons per $\mathrm{g}$ organic carbon (Tissot, et al., 1974). The decrease in $\mathrm{S}_{2}$ values from the darker base to the lighter top of the cycle, therefore, suggests a change from more to less lipid-rich organic matter upward in the cycle. Although there appears to be differences in type of organic matter within individual cycles, shipboard and shorebased RockEval studies and the shorebased studies of humic acids show that most of the organic matter at Site 532 is derived from marine sources (Meyers, Brassell, and Huc, this volume).

\section{Mass Accumulation Rates}

We calculated mass accumulation rates (MAR; g/ $\mathrm{cm}^{2} / \mathrm{m}$.y.) for each 0.5 m.y. interval in order to eliminate the effects of compaction and to examine the influx rates of bulk sediment, $\mathrm{CaCO}_{3}$, biogenic silica, organic 
Table 1. (Continued.)

\begin{tabular}{|c|c|c|c|c|c|c|c|c|c|c|c|c|c|}
\hline Depth-m & $\% \mathrm{CaCO}$ & Depth-m & $2 \mathrm{CaCO}$ & \begin{tabular}{|l|} 
Depth-m \\
\end{tabular} & $2 \mathrm{CaCO} 3$ & Depth-m & $2 \mathrm{CaCO} 3$ & \begin{tabular}{|l|} 
Depth-m \\
\end{tabular} & $2 \mathrm{CaCO} 3$ & Depth-m & Z CaCO3 & Depth-m & $2 \mathrm{CaCO} 3$ \\
\hline 128.84 & $21.0 \bullet$ & 137.31 & $39.0 \bullet$ & 145.49 & 50.1 & 154.45 & $57.0 \bullet$ & 162.90 & 36.9 & 171.81 & $58.0 \bullet$ & 181.10 & 47.2 \\
\hline 129.10 & 28.8 & 137.50 & 43.6 & 145.80 & 37.3 & 154.60 & 57.6 & 163.10 & 44.3 & 171.89 & $54.0 \bullet$ & 181.30 & 47.7 \\
\hline 129.30 & 31.2 & 137.70 & 52.8 & 146.00 & 45.1 & 154.80 & 53.4 & 163.30 & 47.9 & 171.90 & 55.9 & 181.60 & 44.8 \\
\hline 129.50 & 16.1 & 137.90 & 57.0 & 146.20 & 37.8 & 155.00 & 52.8 & 163.50 & 41.5 & 172.10 & 48.5 & 181.76 & $30.0 \bullet$ \\
\hline 129.54 & $12.0 \bullet$ & 138.10 & 60.5 & 146.30 & $43.0 \bullet$ & 155.20 & 48.1 & 163.70 & 48.9 & 172.30 & 48.1 & 181.80 & \\
\hline 129.70 & 24.9 & 138.40 & 55.5 & 146.40 & 46.2 & 155.49 & 47.6 & 164.00 & 45.1 & 172.48 & 40.0 & 182.00 & 42.8 \\
\hline 129.90 & 39.9 & 138.60 & 60.1 & 146.60 & 54.9 & 155.70 & 35.4 & 164.20 & 39.8 & 172.50 & $34.0 \bullet$ & 182.20 & 52.9 \\
\hline 130.10 & 44.0 & 138.61 & $59.0 \bullet$ & 146.80 & 42.0 & 155.80 & $29.0 \bullet$ & 164.40 & 53.8 & 172.80 & 54.6 & 182.40 & 52.9 \\
\hline 130.29 & 38.9 & 138.80 & 57.3 & 147.00 & $53.0 \bullet$ & 155.90 & 45.6 & 164.55 & $51.0 \bullet$ & 173.00 & 54.5 & 182.50 & $53.0 \bullet$ \\
\hline 130.58 & $46.0 \bullet$ & 139.00 & 51.1 & 147.20 & 51.8 & 156.10 & 46.2 & 164.60 & 53.2 & 173.21 & 47.7 & 182.60 & 52.5 \\
\hline 130.60 & 48.0 & 139.20 & 42.4 & 147.31 & 53.0 & 156.30 & 47.2 & 164.80 & 51.1 & 173.40 & 43.1 & 182.80 & $55.0 \bullet$ \\
\hline 130.80 & 45.1 & 139.40 & 48.0 & 147. & & 156 & 47.7 & 1 & & 173.60 & 37.8 & & 63.2 \\
\hline 131.00 & 27.3 & 139.40 & 50.4 & 147 & 0 & 156. & 48.1 & 16 & 42.4 & 173 & 48.0 & 0 & 62.7 \\
\hline 131.20 & 36.2 & 139.60 & 52.3 & 147.80 & 55.5 & 156.94 & 55.7 & 165.40 & 43.5 & 174.20 & 44.9 & 183.40 & 61.6 \\
\hline 131.40 & 39.5 & 139.80 & 38.3 & 148.00 & 54.3 & 157.00 & 52.8 & 165.60 & 50.1 & 174.40 & 46.4 & 183.60 & 60.7 \\
\hline 131.91 & $20.0 \bullet$ & 140.00 & 36.4 & 148.30 & 55.0 & 157.20 & 52.3 & 165.80 & 45.7 & 174.60 & 47.8 & 183.80 & 53.3 \\
\hline 132.00 & 9.2 & 140 & $\begin{array}{l}30.4 \\
46.9\end{array}$ & 148.50 & 49.1 & 157. & 48 & 166. & 47.1 & 174.71 & $50.0 \bullet$ & & \\
\hline 132.20 & 27.4 & 140. & 55.4 & 148.70 & 42.9 & 157 & 47.5 & 166.20 & 45.5 & 174.80 & 48.7 & 184 & 48.4 \\
\hline 132.20 & 60.0 & 140.41 & $53.0 \bullet$ & 148.90 & 28.9 & 158.00 & 57.1 & 16 & 44.3 & 175 & 43.3 & 184 & 56.7 \\
\hline 132.40 & 25.4 & 140.60 & 54.6 & 149.10 & 44.6 & 158.20 & 55.6 & 166.60 & 44.5 & 175.17 & $38.0 \bullet$ & 184.50 & 67.4 \\
\hline 132.60 & 33.0 & 140.90 & 50.8 & 149.30 & 38.7 & 158.50 & 56.7 & 166.84 & 34.2 & 175.20 & 37.4 & 184.70 & 71.1 \\
\hline 132.80 & 42.2 & 141.10 & 37.4 & 149.50 & 32.6 & 158.70 & 45.1 & 167.10 & 49.8 & 175.40 & 48.6 & 184.72 & $68.0 \bullet$ \\
\hline 133.00 & 50.9 & 141.19 & $33.0 \bullet$ & 149.80 & 41.6 & 158.90 & $45.0 \bullet$ & 167.30 & 51.0 & 175.70 & 53.9 & 184.90 & 71.2 \\
\hline 133.20 & 44.4 & 141.30 & 39.4 & 150.00 & 43.3 & 158.91 & 47.1 & 167.50 & 52.5 & 175.90 & 61.0 & 187.00 & 63.2 \\
\hline 133.50 & 34.5 & 141.41 & $25.0 \bullet$ & 150.20 & 51.3 & 159.10 & 51.4 & 167.70 & 45.4 & 176.10 & 54.3 & 187.20 & 52.3 \\
\hline 1. & $33.9 \bullet$ & 141.50 & 45.4 & 150.44 & 53.3 & 159.30 & 56.4 & 6 & 39 & 176.33 & 52.2 & 187 & GL \\
\hline & 26. & 141. & & 15 & 54. & 159 & 63 & 168 & 28 & & 43. & & \\
\hline & 37.7 & 141.90 & 42.6 & i & 44.6 & 159.68 & 57.9 & 168 & $27.0 \bullet$ & 17 & 48.6 & 187 & $73.0 \bullet$ \\
\hline 134.10 & 36.3 & 142.10 & 44.3 & 151.00 & $54.0 \bullet$ & 160.00 & 63.1 & 168.40 & 39.9 & 177.60 & 45.4 & 187.81 & 72.9 \\
\hline 134.30 & 50.5 & 142.29 & 51.8 & 151.20 & 61.9 & 160.20 & 58.1 & 168.60 & 49.4 & 177.82 & 45.5 & 188.00 & 73.0 \\
\hline 134.50 & 46.0 & 142.44 & 61.8 & 151.42 & 51. & 160.40 & 57.7 & 168.80 & 46. & 178.00 & 43.8 & 188.20 & 61.4 \\
\hline 134. & 59.3 & 142. & & & 59 & 160 & 60 & 169 & 48 & & 44. & & 60.9 \\
\hline 134.77 & $62.0 \bullet$ & 143. & & & 54.0 & 160. & 68. & 169 & 45 & 178 & 34.7 & & 48.4 \\
\hline 135. & $60: 4$ & 143. & 37.5 & & 53.6 & 161.00 & 56.1 & 169 & 48.8 & & 36.6 & & 52.9 \\
\hline 135.20 & 45.1 & 143.40 & 37.8 & 152.00 & 48.5 & 161.00 & 69.9 & 169.43 & 53.2 & 178.80 & 42.1 & 189.10 & 37.9 \\
\hline 135.40 & 56.7 & 143.60 & 40.4 & 1 & 43. & 161.2 & 56.5 & 169 & 53.6 & 179 & 38.1 & 189.30 & $44.0 \bullet$ \\
\hline & & & & & 40.0 & & 66 & & 52 & & 44 & & 48.0 \\
\hline 13 & & 144. & 3 & is & 41.6 & 161. & 43.1 & & 58.4 & & 49. & & 48.4 \\
\hline 136.00 & 58.7 & 144.30 & & 152.70 & 44.8 & 161.55 & $37.0 \bullet$ & 170.40 & 52.9 & 179.60 & 44.5 & 189.67 & 53.2 \\
\hline 136.20 & 55.8 & 144.50 & 51.0 & 152.90 & 53.8 & 161.60 & 41.5 & 170.60 & 54.4 & 179.80 & 42.2 & 190.00 & 53.2 \\
\hline 136.40 & 55. & 1 & 57.7 & 1 & 3 & & 53 & 170. & 51.8 & 180 & 40.0 & 19 & 49.6 \\
\hline & & & & & & & & & & & & & \\
\hline & & & & & 44 & & 51 & & & & & & 57 \\
\hline 137.10 & 39.6 & 145.10 & 53.2 & & 48.8 & & 49.5 & & 49.2 & & 52.3 & & $55.0 \bullet$ \\
\hline 137.30 & 39.6 & 145.30 & 47.2 & 154.40 & 56.6 & 162.70 & 46.2 & 171.70 & 51.9 & 180.90 & 46.4 & 190.80 & 55.9 \\
\hline
\end{tabular}

carbon, and clay (Table 5). Average accumulation rates were calculated for each time interval (length of section in $\mathrm{m} / 0.5 \mathrm{~m} . \mathrm{y}$.), and the average dry bulk density for each interval was calculated from porosity data (Site 532 , this volume) according to the equation:

Dry bulk density $(\mathrm{DBD})=(1-$ porosity $/ 100) 2.7$,

where 2.7 is the assumed average grain density (Rea and Janecek, 1981). Values of DBD are in $\mathrm{g} / \mathrm{cm}^{3}$, and if multiplied by 100 are in units of $\mathrm{g} / \mathrm{cm}^{2} / \mathrm{m}$ of section. Values of $\mathrm{DBD} / \mathrm{m}$ of section, when multiplied by the average accumulation rates in $\mathrm{m} / \mathrm{m}$.y. give MAR values for bulk sediment in units of $\mathrm{g} / \mathrm{cm}^{2} / \mathrm{m}$.y. (Table 5). The overall equation, therefore, for calculating bulk sediment MAR is:

$$
\begin{gathered}
\text { Bulk sediment } \operatorname{MAR}\left(\mathrm{g} / \mathrm{cm}^{2} / \mathrm{m} . \mathrm{y} .\right)= \\
(\text { accumulation rate }) \times 100 \times \\
(1-\text { porosity } / 100) \times 2.7
\end{gathered}
$$

An alternate method of obtaining DBD is to use the wet-bulk density (WBD) obtained from shipboard gam- ma-ray attenuation porosity evaluator (GRAPE) relationship between DBD and WBD is:

$$
\mathrm{DBD}=\mathrm{WBD}-(0.01025 \times \text { porosity })
$$

(van Andel et al., 1975). Both methods involve assumptions: Equation (1) assumes a grain density of $2.7 \mathrm{~g} / \mathrm{cm}^{3}$ and Equation (3) relies on GRAPE data. Because of the uncertainties in GRAPE data, we chose to use MAR values calculated by Equation (1).

The next step was to determine the MARs for individual sediment components using average measured percentages of $\mathrm{CaCO}_{3}$, organic carbon, and biogenic silica (opal) for each interval in Table 5. Average concentrations of $\mathrm{CaCO}_{3}$ and organic carbon for each 0.5 m.y. interval were taken from Table 3 .

Data for percent biogenic silica (percent opal) were obtained by two different methods. First, the total percentages of siliceous biogenic components were taken from smear-slide estimates (Fig. 2; Site 532 summary chapter, this volume) and averaged for each interval. The second estimation of biogenic silica was obtained from analyses of $\mathrm{SiO}_{2}$ and $\mathrm{Al}_{2} \mathrm{O}_{3}$ by X-ray fluorescence 
Table 1. (Continued.)

\begin{tabular}{|c|c|c|c|c|c|c|c|c|c|c|c|}
\hline Depth-m & \% $\mathrm{CaCO} 3$ & Depth-m & $\% \mathrm{CaCO} 3$ & Depth-m & $\% \mathrm{CaCO} 3$ & Depth-m & $x \mathrm{CaC} 03$ & Depth-m & $\approx \mathrm{CaCO} 3$ & Depth-m & $2 \mathrm{CaCO} 3$ \\
\hline 191.25 & 53.9 & 199.80 & 53.6 & 212.00 & 71.1 & 225.09 & $64.0 \bullet$ & 234.80 & 61.3 & 247.30 & 50.4 \\
\hline 191.40 & 50.7 & 200.00 & 56.4 & 212.20 & 65.2 & 225.20 & 64.4 & 235.00 & 63.0 & 247.49 & 48.9 \\
\hline 191.60 & 53.1 & 200.20 & 53.3 & 212.24 & $44.0 \bullet$ & 225.40 & 66.3 & 235.00 & 64.7 & & \\
\hline 191.80 & 64.0 & 200.30 & $55.0 \bullet$ & 212.40 & 56.2 & 225.70 & 66.2 & 235.17 & 58.2 & & \\
\hline 192.10 & 61.0 & 200.40 & 52.4 & 212.60 & 60.6 & 225.90 & 66.7 & 235.20 & 55.9 & & \\
\hline 192.30 & 55.6 & 200.60 & 52.0 & 212.80 & 58.2 & 226.10 & 63.2 & 235.40 & 63.2 & & \\
\hline 192.50 & 54.9 & 200.81 & 50.4 & 213.00 & 66.5 & 226.30 & 66.8 & 235.47 & $60.0 \bullet$ & & \\
\hline 192.59 & $56.0 \bullet$ & 201.00 & 50.6 & 213.30 & 62.9 & 226.50 & 54.1 & 235.60 & 60.5 & & \\
\hline 192.70 & 56.5 & 201.30 & 54.0 & 213.50 & 59.2 & 226.59 & $48.0 \bullet$ & 235.81 & 66.9 & & \\
\hline 192.90 & 54.0 & 201.50 & 61.9 & 213.70 & 58.1 & 226.71 & 46.6 & 236.00 & 68.4 & & \\
\hline 193.10 & 54.7 & 201.70 & 62.6 & 213.90 & 63.5 & 226.90 & 53.1 & 236.20 & 66.6 & & \\
\hline 193.30 & 57.1 & 201.90 & 55.7 & 214.10 & 54.4 & 227.20 & 54.5 & 236.50 & 61.7 & & \\
\hline 193.60 & 61.1 & 201.96 & $55.0 \bullet$ & 214.30 & 50.1 & 227.40 & 53.2 & 236.70 & 57.1 & & \\
\hline 193.80 & 62.3 & 202.80 & 59.4 & 214.51 & 48.5 & 227.60 & 57.2 & 236.90 & 40.9 & & \\
\hline 194.00 & 57.2 & 203.00 & 54.5 & 214.80 & 58.7 & 228.20 & 54.6 & 236.97 & $32.0 \bullet$ & & \\
\hline 194.20 & 56.3 & 203.20 & 59.2 & 215.00 & 51.4 & 228.40 & 51.8 & 237.12 & 37.0 & & \\
\hline 194.39 & 53.9 & 203.40 & 52.6 & 215.20 & 54.1 & 228.60 & 62.8 & 237.31 & 43.6 & & \\
\hline 194.50 & $41.0 \bullet$ & 203.60 & 51.7 & 215.40 & 58.0 & 228.80 & 61.4 & 238.06 & 45.7 & & \\
\hline 194.59 & 49.7 & 203.80 & 40.5 & 215.60 & 56.4 & 229.00 & 62.4 & 238.25 & 49.0 & & \\
\hline 194.80 & 50.2 & 203.80 & 51.3 & 215.78 & 55.7 & 229.20 & 65.1 & 238.60 & 55.0 & & \\
\hline 195.10 & 54.3 & 204.00 & 56.7 & 220.20 & 67.4 & 229.40 & 65.5 & 238.80 & 54.5 & & \\
\hline 195.30 & 60.9 & 204.20 & 46.9 & 220.40 & 69.7 & 229.70 & 52.9 & 239.00 & 58.3 & & \\
\hline 195.50 & 61.7 & 204.42 & $46.0 \bullet$ & 220.60 & 71.1 & 229.84 & $47.0 \bullet$ & 239.20 & 56.3 & & \\
\hline 195.80 & 36.2 & 204.43 & 21.0 & 220.80 & 70.2 & 229.90 & 48.9 & 239.48 & 53.5 & & \\
\hline 196.00 & 51.3 & 204.60 & 18.2 & 220.81 & $47.0 \bullet$ & 230.10 & 61.4 & 243.00 & 40.1 & & \\
\hline 196.20 & 62.0 & 207.80 & 57.3 & 221.00 & 70.5 & $230 \cdot 30$ & 63.8 & 243.20 & 41.7 & & \\
\hline 196.24 & $62.0 \bullet$ & 208.00 & 43.6 & 221.20 & 62.7 & 230.50 & 60.3 & 243.40 & 47.2 & & \\
\hline 196.40 & 62.5 & 208.20 & 42.5 & 221.40 & 69.8 & 230.70 & 60.8 & 243.60 & 46.9 & & \\
\hline 196.60 & 61.2 & 208.40 & 45.9 & 221.70 & 65.9 & 230.90 & 68.3 & 243.80 & $48.0 \bullet$ & & \\
\hline 196.80 & 55.3 & 208.60 & 45.0 & 221.90 & 59.9 & 231.20 & 69.4 & 244.00 & 43.4 & & \\
\hline 197.00 & 52.3 & 208.80 & 46.4 & 222.10 & 67.9 & 231.40 & 71.9 & 244.20 & 43.4 & & \\
\hline 197.30 & 47.2 & 209.00 & 47.0 & 222.30 & 70.1 & 231.60 & 64.4 & 244.51 & 45.0 & & \\
\hline 197.50 & 50.0 & 209.04 & $61.0 \bullet$ & 222.50 & 74.9 & 231.79 & 64.2 & 244.67 & 48.6 & & \\
\hline 197.68 & 59.6 & 209.30 & 48.4 & 222.70 & 73.4 & 232.00 & 65.5 & 244.72 & 48.0 & & \\
\hline 197.90 & 66.7 & 209.50 & 55.7 & 222.90 & 68.4 & 232.20 & 62.6 & 244.90 & 49.1 & & \\
\hline 198.10 & 59.9 & 209.70 & 64.2 & 223.20 & 70.0 & 232.40 & 58.9 & 245.10 & 51.2 & & \\
\hline 198.30 & 59.5 & 209.85 & $63.0 \bullet$ & 223.40 & 67.6 & 232.49 & $49.0 \bullet$ & 245.30 & 48.6 & & \\
\hline 198.50 & 42.0 & 209.90 & 64.1 & 223.60 & 66.7 & 232.60 & 46.1 & 245.50 & 45.4 & & \\
\hline 198.52 & $34.0 \bullet$ & 210.10 & 62.7 & 223.80 & 66.1 & 232.82 & 59.8 & 245.70 & 46.9 & & \\
\hline 198.65 & $33.0 \bullet$ & 210.30 & 68.2 & 224.00 & 65.3 & 233.10 & 63.5 & 246.00 & 49.1 & & \\
\hline 198.80 & 46.8 & 210.49 & 65.3 & 224.20 & 65.5 & 233.30 & 60.8 & 246.07 & $49.0 \bullet$ & & \\
\hline 199.00 & 55.6 & 210.80 & 60.0 & 224.40 & 68.2 & 233.50 & 65.1 & 246.20 & 51.5 & & \\
\hline 199.20 & 52.6 & 210.99 & 36.0 & 224.60 & 66.5 & 233.69 & $62.0 \bullet$ & 246.43 & 49.6 & & \\
\hline 199.40 & 59.9 & 211.20 & 32.4 & 224.80 & 69.6 & 233.71 & 60.9 & 246.60 & 49.2 & & \\
\hline 199.60 & 51.5 & 211.80 & 66.4 & 225.00 & 67.4 & 234.60 & 65.6 & 246.97 & 51.4 & & \\
\hline
\end{tabular}

- = Shipboard $\mathrm{CaCO}_{3}$ Bomb Analysis

(XRF) (Dean and Parduhn, this volume). A plot of the $\mathrm{SiO}_{2}: \mathrm{Al}_{2} \mathrm{O}_{3}$ ratio (Fig. 2) shows that there is a baseline low of about 3.3 in those parts of the section where no siliceous biogenic debris was detected in smear slides (Fig. 2). We assumed therefore that an average $\mathrm{SiO}_{2}$ : $\mathrm{Al}_{2} \mathrm{O}_{3}$ ratio of 3.3 is representative of nonbiogenic alumniosilicates that were deposited at Site 532. Nonbiogenic $\mathrm{SiO}_{2}$ was then calculated according to the equation:

$$
\text { Nonbiogenic } \mathrm{SiO}_{2}=\left(\% \mathrm{Al}_{2} \mathrm{O}_{3}\right) \times 3.3
$$

Biogenic $\mathrm{SiO}_{2}$ was calculated according to the equation:

$$
\text { Biogenic } \mathrm{SiO}_{2}=\left(\text { total } \mathrm{SiO}_{2}\right)-\left(\text { nonbiogenic } \mathrm{SiO}_{2}\right) \text {. }
$$

Bostrom et al. (1972) used the same method for calculating opaline silica in sediments from the South Atlantic, but assumed a $\mathrm{SiO}_{2}: \mathrm{Al}_{2} \mathrm{O}_{3}$ ratio of 3.1, that of average continental crust. Both smear-slide data and XRF-derived biogenic silica show similar trends (Fig. 2), but the smear-slide estimates usually are higher. We prefer the estimates calculated from the XRF data because smear-slide data are volume estimates that are semiquantitative at best and usually tend to overestimate percentages of siliceous biogenic components, particularly if volume estimates of this high-porosity component are then used for weight-percent calculations.

The component MARs were calculated according to the equation:

$$
\begin{aligned}
\text { Component } \mathrm{MAR} & =\text { (bulk sediment component MAR) } \\
& \times \text { (fraction of component) }
\end{aligned}
$$

The clay MAR for each interval was calculated by the difference between the bulk-sediment MAR for the interval minus the sum of MARs for $\mathrm{CaCO}_{3}$, biogenic $\mathrm{SiO}_{2}$, and organic carbon. Component MARs are tabulated for each 0.5 m.y. interval in Table 5. The MARs are plotted versus time in Figure 8 in order to show general trends in MAR for each component with time. The individual MARs will be discussed in the section on upwelling intensity and organic productivity. 
Table 2. Organic carbon data, Site 532.

\begin{tabular}{|c|c|c|c|c|c|c|c|c|c|c|c|c|c|}
\hline Depth-m & z c-org. & Depth-m & $z \mathrm{C}$-org. & vepth-m & $z \mathrm{c}$-ork. & Depth-a & z C-org. & vepth-m & $z$ c-org. & Depth-m & $\mathrm{zC}$-org. & Depth-m & $x \cos x$. \\
\hline .20 & 1.63 & 10.10 & 2.57 & 18.40 & $4.9 b$ & 28.10 & 4.39 & 37.50 & 1.72 & 46.10 & 3.38 & 55.60 & 2.44 \\
\hline .40 & 3.38 & 10.20 & .990 & 18.60 & 7.43 & 28.30 & 4.61 & 37.70 & 1.90 & 46.30 & 5.82 & 55.80 & 2.97 \\
\hline .60 & 2.10 & 10.30 & 1.52 & 18.90 & 3.70 & 28.50 & 5.06 & 38.00 & 2.25 & 46.50 & 5.30 & 56.00 & 4.69 \\
\hline .80 & 2.77 & 10.50 & 2.13 & 19.10 & 5.48 & 28.70 & 3.91 & 38.20 & 1.72 & 46.70 & 6.510 & 56.20 & 3.20 \\
\hline 1.00 & 4.70 & 10.70 & 1.73 & 19.30 & 4.99 & 28.90 & 5.32 & 38.40 & 2.88 & 46.80 & 3.64 & 56.30 & 2.650 \\
\hline 1.20 & 3.78 & 10.90 & 2.12 & 19.50 & 5.89 & 29.20 & 3.48 & 38.60 & 2.20 & 47.01 & 2.19 & 56.40 & 3.15 \\
\hline 1.40 & 4.10 & 11.10 & 3.40 & 19.70 & 5.39 & 29.40 & 3.82 & 38.80 & 1.75 & 47.13 & $5.52 \bullet$ & 56.60 & 2.42 \\
\hline 1.57 & $3.666^{\circ}$ & 11.30 & 2.39 & 19.90 & 4.66 & 29.60 & 3.58 & 39.00 & 1.20 & 47.20 & 2.47 & 56.77 & .99 \\
\hline 1.70 & 3.67 & 11.40 & 3.28 & 20.10 & 6.05 & 29.80 & 5.16 & 39.03 & $1.16 \bullet$ & 47.40 & 2.63 & 56.80 & 1.520 \\
\hline 1.90 & 4.82 & 11.60 & 2.93 & 20.40 & 4.42 & 30.60 & 4.42 & 39.20 & 2.28 & 47.60 & 3.65 & 57.00 & 4.68 \\
\hline 2.10 & 5.25 & 11.80 & 2.64 & 20.60 & 2.86 & 30.75 & 5.66 & 39.40 & 3.50 & 47.80 & 6.04 & 57.20 & 3.84 \\
\hline 2.30 & 4.29 & 12.00 & 3.00 & 20.80 & 1.68 & 30.80 & 4.72 & 39.46 & 2.590 & 48.00 & 6.42 & 57.40 & 4.72 \\
\hline 3.20 & 4.02 & 12.20 & 2.25 & 21.00 & 1.30 & 31.00 & 4.11 & 39.60 & 1.75 & 48.09 & $1.88 \bullet$ & 57.60 & 3.27 \\
\hline 3.40 & 3.27 & 12.40 & 2.46 & 21.16 & $1.98^{\circ}$ & 31.20 & 4.89 & 39.80 & 1.79 & 48.20 & 3.94 & 57.80 & 2.82 \\
\hline 3.60 & 2.94 & 12.46 & $3.28 \bullet$ & 21.20 & 2.05 & 31.40 & 4.48 & 40.00 & 2.20 & 48.30 & 2.48 & 58.00 & 4.20 \\
\hline 3.80 & 3.22 & 12.60 & 4.32 & 21.40 & 2.63 & 31.60 & 4.10 & 40.20 & 2.22 & 48.40 & 3.26 & 58.20 & 7.61 \\
\hline 4.00 & 3.26 & 12.80 & 2.40 & 21.60 & 2.82 & 31.80 & 3.26 & 40.40 & 2.66 & 48.44 & 6.59 & 58.51 & 7.61 \\
\hline 4.20 & 3.40 & 13.00 & 1.66 & 21.80 & 4.01 & 32.10 & 4.46 & 40.60 & 2.98 & 48.60 & 2.67 & 58.68 & $5.91 \bullet$ \\
\hline 4.40 & 3.13 & 13.04 & 2.45 & 22.00 & 3.59 & 32.30 & 5.15 & 40.90 & 4.18 & 48.80 & 2.98 & 58.70 & 6.47 \\
\hline 4.60 & 3.18 & 13.20 & 3.78 & 22.20 & 2.75 & 32.50 & 2.03 & 41.10 & 2.05 & 49.20 & 1.69 & 58.90 & 5.76 \\
\hline 4.80 & 3.23 & 13.40 & 2.30 & 22.40 & 3.35 & 32.60 & $1.70 \bullet$ & 41.30 & 1.38 & 49.40 & 1.46 & 59.10 & 7.23 \\
\hline 5.00 & 3.54 & 13.60 & 2.27 & 22.60 & 3.21 & 32.70 & 3.07 & 41.34 & $1.26 \bullet$ & 49.60 & 2.60 & 59.30 & 3.68 \\
\hline 5.20 & 3.28 & 13.80 & 2.22 & 22.80 & 2.77 & 32.90 & 2.92 & 41.50 & 1.97 & 49.81 & 2.47 & 59.50 & 3.92 \\
\hline 5.40 & 3.12 & 14.00 & 3.24 & 23.00 & 4.38 & 33.10 & 3.61 & 41.70 & 1.90 & 50.00 & 4.67 & 59.70 & 5.57 \\
\hline 5.70 & 3.84 & 14.20 & 2.42 & 23.30 & 5.90 & 33.30 & 3.80 & 41.80 & $2.20 \bullet$ & 50.20 & 3.91 & 60.10 & 4.00 \\
\hline 5.90 & 3.13 & 14.50 & 1.95 & 23.50 & 5.68 & 33.35 & $3.64 \bullet$ & 41.90 & 1.93 & 50.30 & $5.04 \bullet$ & 60.28 & $2.68^{\circ}$ \\
\hline 6.10 & 2.77 & 14.70 & 1.48 & 23.70 & $6.06 \bullet$ & 33.60 & 5.02 & 42.10 & 1.82 & 50.40 & 5.46 & 60.30 & 2.62 \\
\hline 6.30 & 3.74 & 14.90 & 2.63 & 23.90 & 4.57 & 33.80 & 5.14 & 42.40 & 2.63 & 50.60 & 5.25 & 60.50 & 2.89 \\
\hline 6.50 & 2.39 & 15.10 & 2.85 & 24.10 & 3.45 & 34.00 & 4.18 & 42.60 & 3.14 & 50.80 & 4.83 & 60.70 & 3.23 \\
\hline 6.70 & 4.02 & 15.30 & 2.58 & 24.30 & 3.05 & 34.20 & 5.33 & 42.80 & 3.13 & 51.10 & 3.59 & 60.90 & 4.29 \\
\hline 6.90 & 5.17 & 15.50 & 2.55 & 24.50 & 2.36 & 34.40 & 4.48 & 43.00 & 3.35 & 51.30 & 4.31 & 61.40 & 3.56 \\
\hline 7.10 & $2.70 \bullet$ & 15.70 & 2.62 & 24.80 & 2.74 & 34.96 & 2.01 & 43.20 & 4.04 & 51.50 & 4.28 & 61.60 & 4.33 \\
\hline 7.20 & 3.41 & 16.00 & 3.21 & 25.00 & 4.34 & 35.00 & 3.65 & 43.60 & $4.30 \bullet$ & 52.60 & 4.45 & 61.80 & 4.38 \\
\hline 7.40 & 2.61 & 16.20 & 2.75 & 25.20 & 4.08 & 35.20 & 2.79 & 43.90 & 4.06 & 52.80 & 4.37 & 62.00 & 4.10 \\
\hline 7.60 & 2.73 & 16.40 & 3.08 & 25.40 & 3.65 & 35.40 & 2.30 & 44.00 & 2.63 & 53.00 & 4.22 & 62.20 & 6.39 \\
\hline 7.80 & 2.700 & 16.60 & 5.02 & 25.60 & 2.55 & 35.60 & 2.42 & 44.20 & 2.56 & 53.09 & 3.48 & 62.40 & 4.57 \\
\hline 8.00 & 3.28 & 16.80 & 3.34 & 25.80 & 3.18 & 35.80 & 2.04 & 44.40 & 4.06 & 53.20 & 3.63 & 62.60 & 4.25 \\
\hline 8.20 & 3.52 & 17.00 & 6.41 & 26.00 & 3.83 & 36.00 & 1.72 & 44.60 & 3.90 & 53.40 & 3.91 & 62.88 & 2.63 \\
\hline 8.60 & 4.04 & 17.18 & 5.32 & 26.20 & 3.91 & 36.20 & 2.34 & 44.80 & 3.18 & 53.60 & 3.56 & 63.00 & 3.450 \\
\hline 8.80 & 4.03 & 17.20 & $4.88 \bullet$ & 26.40 & 3.26 & 36.50 & 2.84 & 45.00 & 2.99 & 53.80 & 3.75 & 63.10 & 3.57 \\
\hline 9.00 & 3.20 & 17.40 & 3.94 & 26.80 & 2.88 & 36.70 & 2.61 & 45.30 & 3.89 & 54.10 & 2.96 & 63.30 & 6.33 \\
\hline 9.20 & 2.84 & 17.60 & 5.30 & 27.00 & 2.23 & 36.90 & 3.68 & 45.50 & 5.01 & 54.30 & 3.40 & 63.50 & 7.61 \\
\hline 9.40 & 3.22 & 17.80 & 5.66 & 27.20 & 2.56 & 37.10 & 2.91 & 45.70 & 2.89 & 54.43 & 2.74 & 63.70 & 6.27 \\
\hline 9.60 & 2.97 & 18.00 & 6.08 & 27.70 & 4.95 & 37.30 & 1.96 & 45.80 & 1.800 & 54.50 & 2.24 & 63.90 & 4.32 \\
\hline 9.80 & 3.69 & 18.20 & 5.85 & 27.90 & 4.22 & 37.38 & $3.57 \bullet$ & 45.90 & 2.15 & 54.70 & 3.12 & 64.10 & 4.72 \\
\hline
\end{tabular}

\section{DISCUSSION}

\section{Origin of the $\mathrm{CaCO}_{3}$ and Color Cycles}

Carbonate cycles can result from $\mathrm{CaCO}_{3}$ dissolution, dilution of $\mathrm{CaCO}_{3}$ by noncarbonate material, or changes in carbonate productivity. Carbonate dissolution is not an important cause of the cycles at Site 532 because the site is only $1331 \mathrm{~m}$ deep, and the calcareous nannofossils are moderate to well preserved with no apparent signs of dissolution (Site 532 summary chapter, this volume). The magnitudes and trends of the periodicities of the $\mathrm{CaCO}_{3}$ cycles at Site $\mathbf{5 3 2}$ are similar to those from the western Caribbean and eastern equatorial Pacific (Gardner, 1982). The similarities of $\mathrm{CaCO}_{3}$ periodicities from such widespread areas suggest that productivity, probably a local effect, is not a controlling factor in the $\mathrm{CaCO}_{3}$ cycles. Dilution of $\mathrm{CaCO}_{3}$ by fine-grained terrigenous material is a possibility for control of the $\mathrm{CaCO}_{3}$ cycles at Site 532 because the area is on the continental margin of Africa. However, dilution by noncarbonate terrigenous material implies fluctuations of eustatic sea level as the probable primary mechanism.
The fact that the darker layers contain more clay than the lighter layers suggests that there was at least some dilution by terrigenous clastics. Also, even though the dark layers usually contain the highest concentrations of biogenic silica, this is not true for all cycles, and smearslide data usually do not show systematic variations in siliceous biogenic debris within cycles. Semiquantitative estimates of clay abundance from smear slides show that clay content of dark interbeds is about $25 \%$ compared with about $10 \%$ in the lightest parts of light-colored, high- $\mathrm{CaCO}_{3}$ interbeds. This difference represents a $150 \%$ increase in clay content between lighter and darker interbeds. A $175 \%$ increase in noncarbonate dilution is needed to reduce the higher concentrations of $\mathrm{CaCO}_{3}$ in dark-colored interbeds. This $175 \%$ calculated dilution of $\mathrm{CaCO}_{3}$ is reasonably close to the semiquantitative smear-slide estimate of clay increase, which suggests that dilution by terrigenous clastics alone could account for the dark interbeds of the $\mathrm{CaCO}_{3}$ cycles.

The range of periodicities of the $\mathrm{CaCO}_{3}$ at Site 532 ( $26 \mathrm{k} . y$. to $49 \mathrm{k} . \mathrm{y}$.) is similar to that of $\mathrm{CaCO}_{3}$ cycles from Neogene and Quaternary sections from the eastern 
Table 2. (Continued.)

\begin{tabular}{|c|c|c|c|c|c|c|c|c|c|c|c|}
\hline Depth-m & z C-org. & Depth-m & z C-org. & Depth-m & z c-org. & Depth-m & z C-org. & Depth-m & \% c-org. & Depth-m & $z$ C-org. \\
\hline 64.39 & 3.48 & 76.70 & 4.90 & 89.39 & $6.35 \bullet$ & 102.00 & 1.26 & 130.60 & 2.260 & 184.70 & .550 \\
\hline 64.50 & 2.010 & 76.81 & 4.30 & 89.40 & 5.900 & 102.50 & .96 & 131.20 & 3.32 & 189.30 & 2.040 \\
\hline 64.61 & 3.58 & 76.90 & 3.39 & 89.50 & 3.07 & 102.90 & 1.23 & 131.91 & $5.86 \bullet$ & 190.80 & 1.750 \\
\hline 64.80 & 4.83 & 77.10 & 4.19 & 89.90 & 3.24 & 103.10 & 1.95 & 132.00 & 7.00 & 195.30 & $2.59 \bullet$ \\
\hline 65.00 & 5.50 & 77.80 & 5.61 & 90.10 & 3.44 & 103.30 & 1.92 & 133.80 & 1.800 & 196.30 & $.93 \bullet$ \\
\hline 65.20 & 4.05 & 78.00 & 4.54 & 90.30 & 3.06 & 103.57 & $2.65 \bullet$ & 135.40 & 1.24 & 198.50 & 3.750 \\
\hline 65.40 & 3.93 & 78.20 & 3.57 & 90.50 & 3.61 & 103.70 & 1.63 & 136.00 & 1.04 & 200.30 & 1.650 \\
\hline 65.60 & 4.23 & 78.40 & 3.00 & 90.80 & 2.21 & 104.00 & 2.38 & 136.60 & 2.02 & 202.00 & .450 \\
\hline 65.75 & 4.11 & 78.60 & 3.09 & 91.00 & 1.96 & 104.20 & 2.26 & 137.30 & 2.980 & 203.60 & 2.020 \\
\hline 65.90 & 3.86 & 80.00 & 2.49 & 91.20 & 2.10 & 104.40 & 3.21 & 137.50 & 1.98 & 204.42 & $2.24 \bullet$ \\
\hline 66.00 & 4.28 & 80.20 & 2.48 & 91.40 & 1.60 & 104.60 & 2.91 & 138.60 & .800 & 204.60 & 3.99 \\
\hline 66.80 & 2.460 & 80.50 & 2.88 & 91.50 & 1.360 & 104.73 & $3.40 \bullet$ & 139.40 & 1.50 & 209.04 & $2.02 \bullet$ \\
\hline 67.00 & 2.01 & 80.70 & 2.87 & 91.60 & 1.78 & 105.40 & 3.69 & 140.40 & .910 & 209.85 & $1.03^{\bullet}$ \\
\hline 67.50 & 3.02 & 80.90 & 2.29 & 91.80 & 1.77 & 105.60 & 6.02 & 140.60 & 1.310 & 211.20 & 4.09 \\
\hline 67.70 & 2.27 & 81.10 & 4.020 & 92.00 & 1.68 & 105.71 & $6.04 \bullet$ & 141.20 & 2.130 & 212.24 & $2.86 \bullet$ \\
\hline 68.30 & 2.65 & 81.30 & 2.47 & 92.30 & 2.05 & 105.80 & 5.83 & 141.41 & $5.540^{\circ}$ & 214.50 & 2.240 \\
\hline 68.30 & $5.00 \bullet$ & 81.49 & 3.34 & 93.20 & 1.69 & 105.98 & 6.22 & 145.00 & 2.170 & 220.81 & $3.95 \bullet$ \\
\hline 68.80 & 4.98 & 81.68 & 2.50 & 93.40 & 2.560 & 106.60 & 2.82 & 146.00 & 2.36 & 229.00 & .680 \\
\hline 69.00 & 2.970 & 82.00 & 2.04 & 94.10 & 2.05 & 106.90 & 1488 & 146.30 & 3.270 & 229.84 & $1.99 \circ$ \\
\hline 69.01 & 4.02 & 82.20 & 2.22 & 94.30 & 2.12 & 107.10 & 2.24 & 147.30 & 2.470 & 232.49 & $2.20 \bullet$ \\
\hline 69.40 & 6.13 & 82.40 & 2.57 & 94.50 & 1.58 & 107.21 & $3.03 \bullet$ & 147.60 & 2.00 & 236.97 & $3.11 \bullet$ \\
\hline 69.80 & 2.69 & 82.60 & 2.55 & 94.70 & 3.43 & 107.50 & 7.65 & 148.70 & 3.87 & 237.20 & 2.060 \\
\hline 70.00 & 5.34 & 82.80 & 2.70 & 94.86 & $4.12 \bullet$ & 107.70 & 6.17 & 149.10 & 2.50 & 242.10 & 1.690 \\
\hline 70.80 & 3.150 & 83.00 & 3.32 & 94.90 & 4.56 & 108.10 & 1.25 & 150.44 & 1.78 & 242.30 & 2.660 \\
\hline 71.40 & 3.13 & 83.60 & 4.56 & 95.20 & 4.91 & 108.30 & 1.77 & 150.60 & 1.94 & 244.72 & $1.23 \bullet$ \\
\hline 71.90 & 3.80 & 83.80 & 4.41 & 95.40 & 6.19 & 108.43 & 1.47 & 152.60 & 2.310 & 250.30 & 1.660 \\
\hline 72.30 & 2.78 & 83.90 & 3.150 & 95.60 & 3.30 & 110.09 & $2.47 \bullet$ & 154.50 & .940 & 250.80 & $1.43 \bullet$ \\
\hline 72.31 & $3.20 \bullet$ & 84.00 & 3.82 & 95.97 & $2.54{ }^{\circ}$ & 111.20 & 1.560 & 154.80 & 2.02 & 254.00 & .570 \\
\hline 72.50 & 2.77 & 84.21 & 4.31 & 96.30 & 2.760 & 114.86 & $3.87 \bullet$ & 155.49 & 2.63 & 257.70 & 2.170 \\
\hline 72.70 & 2.48 & 84.40 & 3.78 & 96.40 & 2.75 & 116.10 & 6.08 & 155.80 & $3.12 \bullet$ & 258.50 & 2.920 \\
\hline 72.90 & 2.47 & 84.60 & 3.11 & 96.80 & 1.55 & 116.16 & $4.48 \bullet$ & 158.90 & 1.310 & 260.20 & 1.840 \\
\hline 73.60 & 2.60 & 84.90 & 3.09 & 97.02 & 2.77 & 116.40 & 1.990 & 159.40 & 3.560 & 262.00 & 1.440 \\
\hline 73.80 & 2.08 & 85.10 & 2.62 & 97.60 & 1.82 & 119.10 & 2.260 & 161.55 & $2.99 \bullet$ & 262.30 & .210 \\
\hline 73.90 & 3.060 & 85.30 & 2.34 & 98.10 & 2.26 & 119.90 & 2.53 & 167.50 & 2.370 & 267.10 & 3.270 \\
\hline 74.00 & 4.44 & 85.51 & 2.59 & 98.50 & 1.87 & 120.10 & 1.78 & 168.24 & $4.11 \bullet$ & 269.10 & 2.150 \\
\hline 74.20 & 4.27 & 86.60 & 4.39 & 98.90 & 2.16 & 120.29 & 3.08 & 171.80 & 1.910 & 270.60 & 2.610 \\
\hline 74.60 & 4.30 & 86.80 & 2.68 & 99.60 & 1.47 & 122.40 & 2.510 & 171.89 & $1.540^{\circ}$ & 272.00 & .680 \\
\hline 74.80 & 4.25 & 86.90 & 4.910 & 99.70 & 4.050 & 122.70 & 2.44 & 172.50 & $2.90 \bullet$ & 273.50 & .380 \\
\hline 75.00 & 5.96 & 87.00 & 3.01 & 99.80 & 1.29 & 123.90 & $4.440^{\circ}$ & 174.70 & 1.160 & 277.20 & 2.110 \\
\hline 75.20 & 4.78 & 87.41 & 2.93 & 100.00 & 1.60 & 128.00 & 2.47 & 175.20 & 3.220 & 277.90 & 2.850 \\
\hline 75.30 & 4.120 & 87.60 & 2.75 & 101.00 & 1.82 & 128.80 & 5.17 & 175.30 & 3.920 & 281.50 & .140 \\
\hline 75.60 & 2.93 & 87.80 & 4.35 & 101.40 & 2.41 & 129.50 & 5.84 & 175.70 & 2.39 & 284.60 & .500 \\
\hline 75.80 & 3.05 & 88.60 & 4.29 & 101.60 & 1.41 & 129.54 & $5.87 \bullet$ & 180.80 & 4.240 & 285.60 & .980 \\
\hline 76.30 & 3.97 & 88.80 & 4.96 & 101.80 & 1.58 & 129.60 & 3.710 & 182.50 & $1.69 \bullet$ & 287.10 & .100 \\
\hline 76.50 & 4.56 & 89.30 & 5.85 & 101.90 & 2.310 & 129.70 & 4.89 & 184.00 & 2.200 & 290.00 & 2.110 \\
\hline
\end{tabular}

Shipboard CHN Analysi

equatorial Atlantic (Dean et al., 1981), Western Caribbean (Gardner, 1982), and eastern equatorial Pacific (Gardner, 1982). Carbonate cycles with periods of about 41 k.y. are common in late Quaternary deep-sea sediment and are almost certainly related in some way to the Earth's orbital cycle of obliquity (tilt of the Earth's axis), which has been suggested as one of the main driving forces behind long-term climatic change (Broecker and van Donk, 1970; Hays, et al., 1976). One of the manifestations of global climate change is variations in global ice volume and, therefore, in changes in global sea level. Studies of fluctuations in the composition of oxygen isotopes in benthic foraminifers from Quaternary deep-sea sediment have related them to fluctuations in global ice volume and hence global sea level (Shackleton, 1967). These studies have been extended into the Pliocene (Prell, 1982), and arguments have been presented that oxygen-isotope fluctuations throughout the Tertiary are related to fluctuations in global ice volumes (Matthews and Poore, 1980; Matthews, in press). Climate-related sedimentary cycles are not unique to the Quaternary, but extend well back into the Tertiary
(Dean et al., 1977 and 1981; Clifton, 1980) Mesozoic (Fischer, 1964; Dean et al., 1977; Arthur and Fischer, 1977; McCave, 1979; Arthur, 1979), and even the Paleozoic (Anderson, 1982). These sedimentary cycles from different geologic periods and from widely scattered areas, but all with somewhat similar periodicities, are most likely coincident with, if not the result of, changes in eustatic sea level.

Furthermore, the $\mathrm{CaCO}_{3}$ cycles at Site 532 are present at the base of the recovered section and continue throughout the section. Changes in periodicity of $\mathrm{CaCO}_{3}$ cycles at Site 532 (Table 4) at first appear to correspond to other changes in sediment character, such as the large pulse of siliceous biogenic debris in the upper Pliocene section or the large decreases in clay and $\mathrm{CaCO}_{3}$ MARs in the mid-Pliocene section (Fig. 8). However, the periodicities of the $\mathrm{CaCO}_{3}$ and organic-carbon cycles at Site 532 are similar in magnitude to the $\mathrm{CaCO}_{3}$ cycles at Sites 502 and 503 in the western Caribbean and eastern equatorial Pacific (Fig. 9). In addition, trends of changes in periodicity with time are also similar at all three sites. The similarities of magnitudes and trends of 


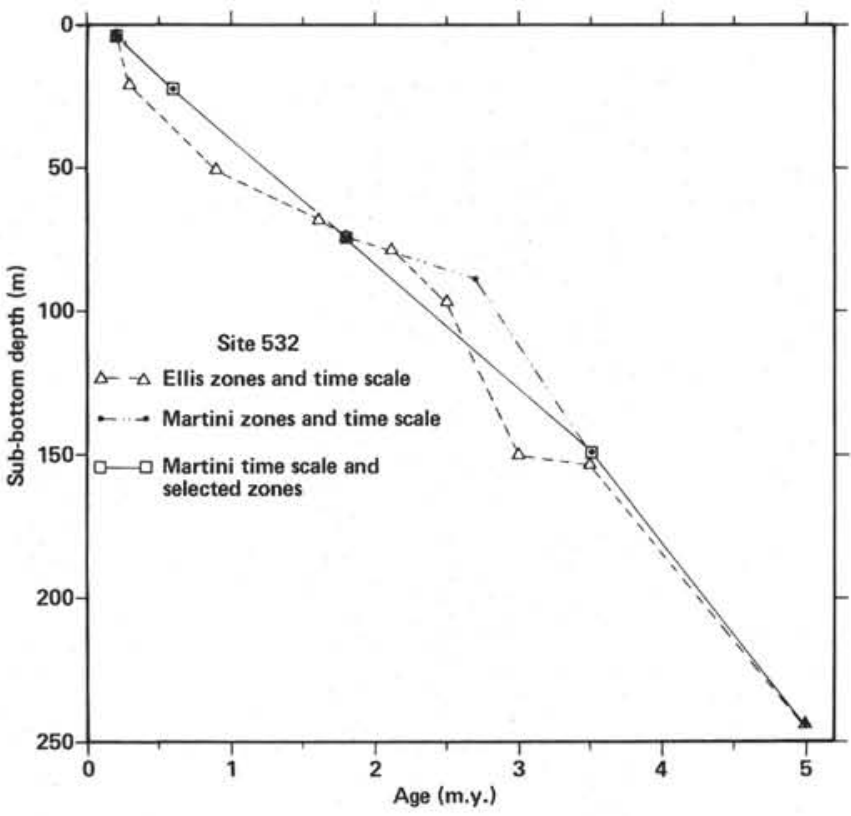

Figure 4. Plots of depth vs. age using the biostratigraphy of Steinmetz (this volume), a modified biostratigraphy with Martini (1980) datums, and our preferred age vs. depth scheme. (See text for explanation.)

Table 3. Statistics for the concentrations of $\mathrm{CaCO}_{3}$, organic carbon, and biogenic $\mathrm{SiO}_{2}$ in Hole 532 for 0.5 -m.y. intervals between 0 and 5.0 m.y. Data for biogenic $\mathrm{SiO}_{2}$ are from Dean and Parduhn (this volume).

\begin{tabular}{|c|c|c|c|c|c|c|c|c|}
\hline \multirow[b]{2}{*}{$\begin{array}{l}0.5 \text { m.y. } \\
\text { intervals }\end{array}$} & \multicolumn{3}{|c|}{$\mathrm{CaCO}_{3}$} & \multicolumn{3}{|c|}{ Organic carbon } & \multicolumn{2}{|c|}{ Biogenic $\mathrm{SiO}_{2}$} \\
\hline & $\begin{array}{l}\text { Number } \\
\text { of } \\
\text { analyses }\end{array}$ & $\begin{array}{l}\text { Mean } \\
(\%)\end{array}$ & $\begin{array}{c}\text { Standard } \\
\text { deviation } \\
(\%)\end{array}$ & $\begin{array}{l}\text { Number } \\
\text { of } \\
\text { analyses }\end{array}$ & $\begin{array}{c}\text { Mean } \\
(\%)\end{array}$ & $\begin{array}{c}\text { Standard } \\
\text { deviation } \\
(\%)\end{array}$ & $\begin{array}{l}\text { Number } \\
\text { of } \\
\text { analyses }\end{array}$ & $\begin{array}{c}\text { Mean } \\
(\%)\end{array}$ \\
\hline $0.0-0.5$ & 85 & 62.4 & 7.1 & 81 & 3.05 & 0.84 & 3 & 0.18 \\
\hline $0.5-1.0$ & 113 & 50.0 & 11.2 & 108 & 3.72 & 1.37 & 6 & 6.62 \\
\hline $1.0-1.5$ & 110 & 38.9 & 11.9 & 110 & 3.56 & 1.43 & 9 & 12.80 \\
\hline $1.5-2.0$ & 117 & 35.0 & 9.0 & 84 & 3.73 & 1.18 & 3 & 9.17 \\
\hline $2.0-2.5$ & 111 & 44.0 & 10.9 & 78 & 2.82 & 1.30 & 4 & 13.30 \\
\hline $2.5-3.0$ & 89 & 36.4 & 10.6 & 27 & 3.54 & 1.86 & 2 & 11.20 \\
\hline $3.0-3.5$ & 117 & 44.6 & 11.2 & 29 & 3.04 & 1.75 & 7 & 3.24 \\
\hline $3.5-4.0$ & 160 & 48,2 & 7.4 & 20 & 2.52 & 1.00 & 6 & 2.43 \\
\hline $4.0-4.5$ & 140 & 54.3 & 9.7 & 17 & 2.11 & 1.10 & 14 & 0.46 \\
\hline $4.5-5.0$ & 130 & 58.0 & 8.9 & 10 & 2.19 & 0.93 & 14 & 3.15 \\
\hline
\end{tabular}

periodicities of $\mathrm{CaCO}_{3}$ cycles from widely separated areas suggest that the $\mathrm{CaCO}_{3}$ cycles are produced by a global, not local, forcing mechanism. The MAR data (Fig. 8) show responses to local conditions such as upwelling and climate on the African continent, but even there local processes probably were triggered by global climatic changes.
The organic-carbon cycles do not appear to be closely related to $\mathrm{CaCO}_{3}$ and color cycles and have a range of periodicities between $28 \mathrm{k} . \mathrm{y}$. and $44 \mathrm{k} . \mathrm{y} . /$ cycle. These periodicities are considerably different from organiccarbon cycles described from the late Neogene and Quaternary from the western Caribbean and eastern equatorial Pacific (Gradner, 1982). The difference in organiccarbon periodicities from widely scattered localities suggests that the forcing mechanism for organic-carbon cycles is a local phenomena and may be diagenetic. The relationships between the trends of average organic carbon and $\mathrm{CaCO}_{3}$ are not entirely independent, however, because the two variables are negatively correlated on scales ranging from individual cycles (Fig. 7) to the entire sequence at Site 532. The correlation coefficient for $\mathrm{CaCO}_{3}$ and organic carbon averaged for $0.5 \mathrm{~m}$.y. intervals (Table 4 ) is -0.62 . Figure 10 shows the smoothed curves of $\mathrm{CaCO}_{3}$ and organic-carbon redrawn from Figure 2, and illustrates the striking negative correlation between $\mathrm{CaCO}_{3}$ and organic carbon at the scale of the $150 \mathrm{k} . y$. cycles for the past $2.5 \mathrm{~m}$.y. Another way of examining the relationship between $\mathrm{CaCO}_{3}$ and organiccarbon is by using a moving correlation coefficient (Dean and Anderson, 1974) the results of which are plotted in Figure 11. Note that there is usually a strong negative correlation between $\mathrm{CaCO}_{3}$ and organic carbon except during periods of strongest upwelling as evidenced by abundance of biogenic $\mathrm{SiO}_{2}$ (compare maximum pulses of biogenic $\mathrm{SiO}_{2}$ in Fig. 2 with zones of nonsignificant correlation coefficients in Fig. 11).

\section{Upwelling Intensity and Organic Productivity}

One of the principal objectives of drilling Site 532 was to recover a detailed late Neogene and Quaternary record of the Benguela-Current upwelling system. Because upwelling intensity, and hence organic productivity, is sensitive to seasonal as well as long-term climatic fluctuations, we first thought that the cycles of $\mathrm{CaCO}_{3}$ and color might be reflections of biogenic silica fluctuations resulting from variations in upwelling intensity. We do not think that this is true, however, for the following reasons. First, diatoms are the dominant biogenic component in sediment from most mid-latitude, open-ocean upwelling areas (Diester-Haass and Schrader, 1979; Schuette and Schrader, 1981; deVries and Schrader, 1981). Siliceous biogenic debris is negligible in the lower Pliocene and upper Quaternary sections at Site 532, and only equal in abundance with nannofossils in the upper Pliocene and lower Quaternary sections

Table 4. Number of cycles and periodicities of $\mathrm{CaCO}_{3}$ and organic carbon for $0.5 \mathrm{~m} . \mathrm{y}$. intervals, Hole 532.

\begin{tabular}{|c|c|c|c|c|c|c|c|c|c|}
\hline \multirow{2}{*}{$\begin{array}{c}\text { Time } \\
\text { interval } \\
\text { (m.y.) }\end{array}$} & \multicolumn{2}{|c|}{$\mathrm{CaCO}_{3}$} & \multicolumn{2}{|c|}{$\mathrm{C}_{\text {org }}$} & \multirow[b]{2}{*}{$\begin{array}{l}\text { Number of } \\
\text { dark beds }\end{array}$} & \multirow[b]{2}{*}{$\begin{array}{l}\text { Periodicity } \\
\text { (k.y./cycle) }\end{array}$} & \multicolumn{3}{|c|}{ Mean values } \\
\hline & $\begin{array}{l}\text { Number } \\
\text { of cycles }\end{array}$ & $\begin{array}{l}\text { Periodicity } \\
\text { (k.y./cycle) }\end{array}$ & $\begin{array}{l}\text { Number } \\
\text { of cycles }\end{array}$ & $\begin{array}{l}\text { Periodicity } \\
\text { (k.y./cycle) }\end{array}$ & & & $\mathrm{CaCO}_{3}$ & $\mathrm{C}_{\text {org }}$ & $\begin{array}{l}\text { Dark beds } \\
\text { (k.y./cycle) }\end{array}$ \\
\hline $0-0.5$ & 14.50 & 34.5 & 18 & 27.8 & 6 & 83.3 & & & \\
\hline $0.5-1.0$ & 14.00 & 35.7 & 18 & 27.8 & 11 & 45.4 & 35,1 & 31.2 & 55.1 \\
\hline $1.0-1.5$ & 15.75 & 31.7 & 15 & 33.3 & 12 & 41.7 & & 31.2 & 33.1 \\
\hline $1.5-2.0$ & 13.00 & 38.5 & 14 & 35.7 & 10 & 50.0 & & & \\
\hline $2.0-2.5$ & 10.25 & 48.8 & 11.5 & 43.5 & 8 & 62.5 & & & \\
\hline $2.5-3.0$ & $10.5 / 425$ k.y. & 40.5 & - & - & $5 / 425$ k.y. & 85.0 & 45.6 & 43.5 & 58.4 \\
\hline $3.0-3.5$ & 10.50 & 47.6 & - & - & 18 & 27.8 & & & \\
\hline $3.5-4.0$ & 19.00 & 26.3 & - & - & 18 & 27.8 & & & \\
\hline $4.0-4.5$ & $16.0 / 410 \mathrm{k} . \mathrm{y}$. & 25.6 & - & - & $14 / 410$ k.y. & 29.3 & 28.4 & - & 29.9 \\
\hline $4.5-5.0$ & $9.0 / 300$ k.y. & 33.3 & - & - & 9/300 k.y. & 33.3 & & & \\
\hline
\end{tabular}



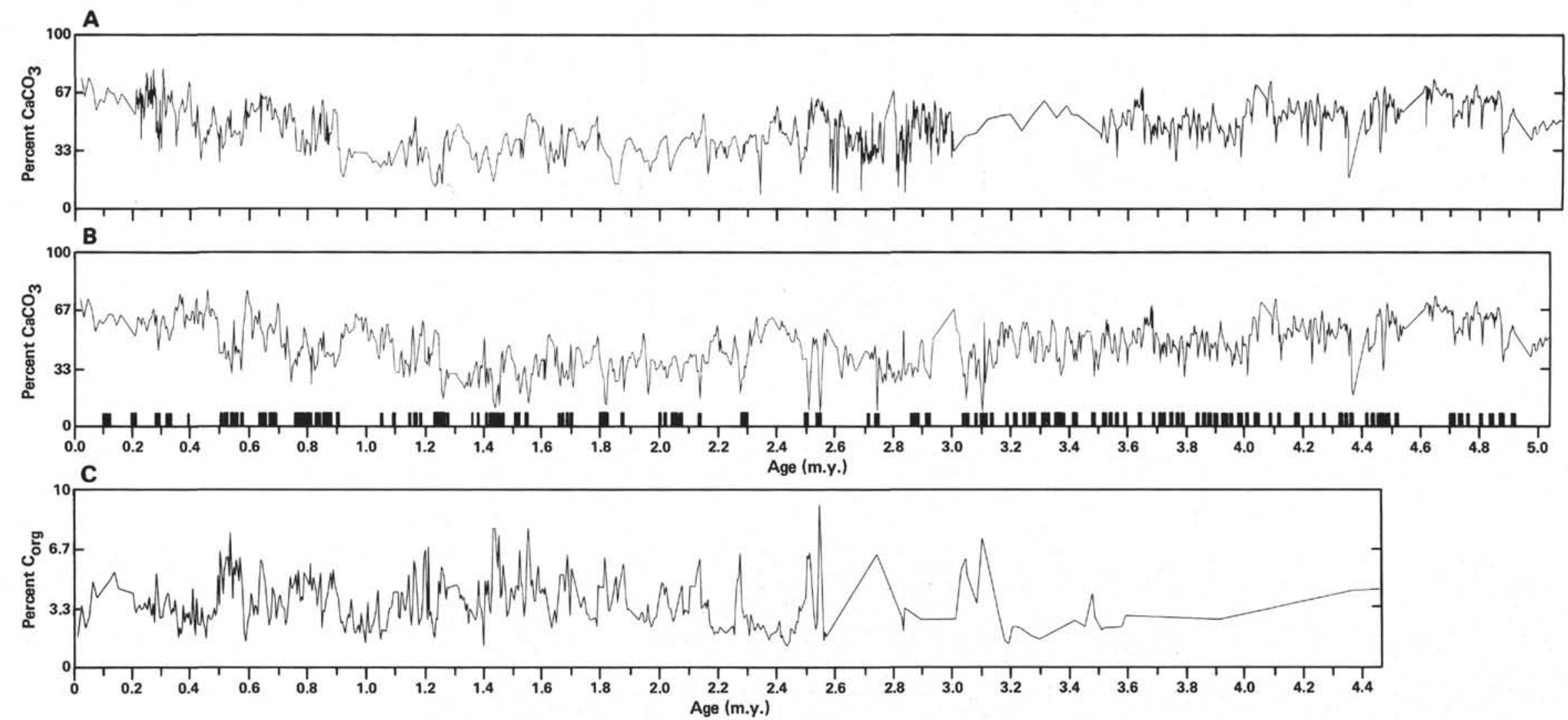

Figure 5. A. Plot of $\mathrm{CaCO}_{3}$ vs. age using the Ellis (1981) nannofossil zonation (Steinmetz and Stradner, this volume). B. Plot of CaCO 3 vs. age for Site 532 using our preferred age model. Black bars along age scale indicate position and thickness of dark-colored sediment. C. Plot of organic carbon vs. age for Site 532 using our preferred age model. (See text for explanation.) 


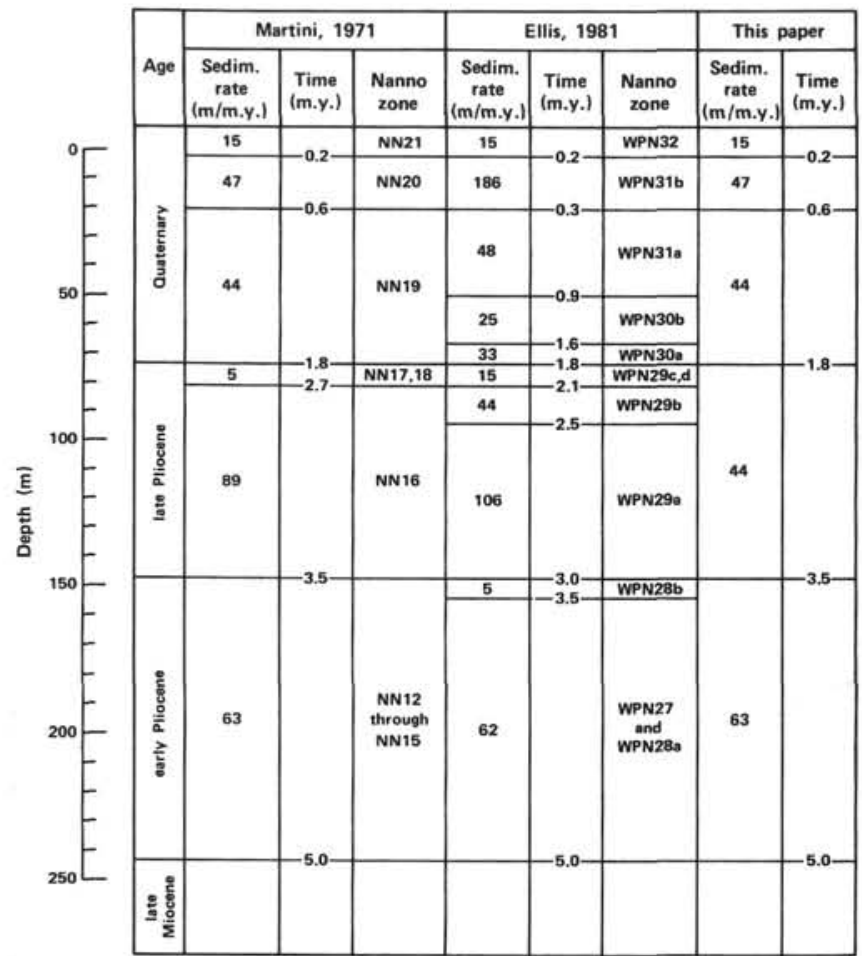

Figure 6. Comparison of zonation and time scale of Martini (1971) with those of Ellis (1981) and the resulting sedimentation rates at Site 532. The zonation, time scale, and sedimentation rates used in this chapter are on the far right. (See text for explanation.)

(Figs. 2 and 8). Also, smear-slide data for the cycle in Core 10 (Fig. 7) and for other cycles (see lithology section in Site 532 summary chapter, this volume) do not show any trends or cyclic changes in diatom abundance that correspond to trends in abundance of $\mathrm{CaCO}_{3}$. Instead, the diatom abundances and biogenic $\mathrm{SiO}_{2}$ MAR abruptly increase in the late Pliocene and early Quaternary (Figs. 2 and 8). This pulse of siliceous biogenic debris, with some relatively high frequency fluctuations, is the strongest evidence in the section to suggest that there were marked changes in the upwelling history at Site 532 .

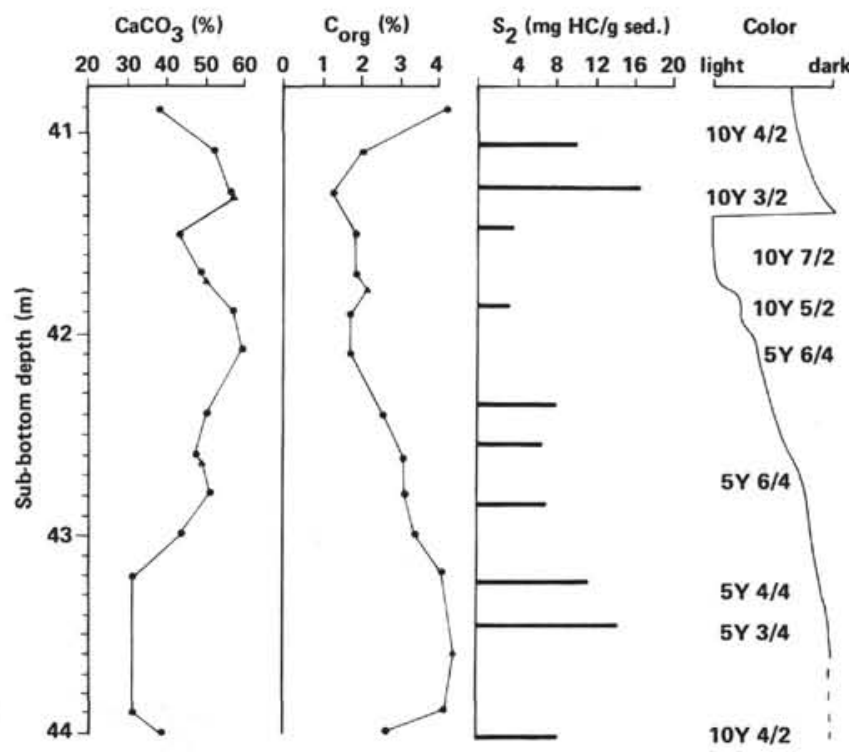

Figure 7. Plots of the data for the dark-light cycle in Hole 532, Core 10 , Sections 2 and 3.

To investigate the broad scale record of upwelling at Site 532 more closely, the curves of MAR (Fig. 8) can be viewed as three segments that represent the pre-upwelling, upwelling, and post-upwelling intervals. The biogenic silica MAR curve was used to define the boundaries between the intervals. Sediment that accumulated during the pre-upwelling interval ( $>3 \mathrm{~m}$.y. old) was a nannofossil marl; $\mathrm{CaCO}_{3}$ and clay both predominated over biogenic silica, each by more than an order of magnitude, and sedimentation rates were relatively high (63 $\mathrm{m} / \mathrm{m} . \mathrm{y}$.). This is not to say, however, that intense upwelling associated with the Benguela Current system did not occur elsewhere during the late Miocene and early Pliocene, perhaps north or south of Site $\mathbf{5 3 2}$ or closer to the south African coast. The center of southwest African coastal upwelling will migrate in response to the location of the main core of the southeast Trade Winds. If the position of the intertropical convergence zone did fluctuate with global climate changes during the late

Table 5. Mass accumulation rates (MAR) for sediment components at Hole 532, using our preferred time scale and zonation. (See text for explanation.)

\begin{tabular}{|c|c|c|c|c|c|c|c|c|c|c|c|c|c|}
\hline $\begin{array}{c}\text { Time } \\
\text { interval } \\
\text { (m.y.) }\end{array}$ & $\begin{array}{c}\text { Depth } \\
\text { interval } \\
\text { (m) }\end{array}$ & $\begin{array}{c}\text { Average } \\
\text { bulk density } \\
\left(\mathrm{g} / \mathrm{cm}^{2}\right)\end{array}$ & $\begin{array}{c}\text { Average } \\
\text { porosity } \\
(\%)\end{array}$ & $\begin{array}{c}\text { Average } \\
\text { water } \\
\text { content } \\
(\%)\end{array}$ & $\begin{array}{c}\text { Average } \\
\text { sediment } \\
\text { rate } \\
(\mathrm{m} / \mathrm{m} . \mathrm{y} .)\end{array}$ & $\begin{array}{c}\text { MAR } \\
\text { bulk } \\
\text { sediment }\end{array}$ & $\begin{array}{c}\text { Average } \\
\mathrm{CaCO}_{3} \\
(\%)\end{array}$ & $\begin{array}{c}\text { MAR } \\
\mathrm{CaCO}_{3}\end{array}$ & $\begin{array}{c}\text { Average } \\
\text { C }_{\text {org }} \\
(\%)\end{array}$ & $\begin{array}{l}\text { MAR } \\
\text { Corg }\end{array}$ & $\begin{array}{c}\text { Average } \\
\text { bio-SiO } \\
(\%)\end{array}$ & $\begin{array}{c}\text { MAR } \\
\text { bio-SiO }\end{array}$ & $\begin{array}{l}\text { MAR } \\
\text { "clay" }\end{array}$ \\
\hline $0-0.5$ & $0-16.9$ & 1.44 & 73 & 52 & 33.8 & 2491 & 62.4 & 1554 & 3.0 & 75 & 0.18 & 4.5 & 858 \\
\hline $0.5-1.0$ & $16.9-39.2$ & 1.47 & 73 & 51 & 44.6 & 3251 & 50.0 & 1626 & 3.7 & 120 & 6.62 & 215 & 1290 \\
\hline $1.0-1.5$ & $39.2-61.2$ & 1.43 & 76 & 55 & 44.0 & 2851 & 38.9 & 1109 & 3.6 & 103 & 12.8 & 365 & 1274 \\
\hline $1.5-2.0$ & $61.2-83.2$ & 1.42 & 79 & 57 & 44.0 & 2495 & 35.0 & 873 & 3.7 & 92 & 9.2 & 230 & 1300 \\
\hline $2.0-2.5$ & $83.2-105.2$ & 1.49 & 75 & 52 & 44.0 & 2970 & 44.0 & 1307 & 2.8 & 83 & 13.3 & 395 & 1185 \\
\hline $2.5-3.0$ & $105.2-127.6$ & 1.50 & 74 & 51 & 44.0 & 3089 & 36.4 & 1124 & 3.5 & 108 & 11.2 & 346 & 1511 \\
\hline $3.0-3.5$ & $127.6-149.6$ & 1.64 & 68 & 42 & 44.0 & 3802 & 44.6 & 1696 & 3.0 & 114 & 3.24 & 123 & 1869 \\
\hline $3.5-4.0$ & $149.6-181.3$ & 1.70 & 65 & 39 & 63.4 & 5991 & 48.2 & 2894 & 2.5 & 150 & 2.43 & 146 & 2801 \\
\hline $4.0-4.5$ & $181.3-213.1$ & 1.71 & 62 & 37 & 63.4 & 6505 & 54.3 & 3532 & 2.1 & 137 & 0.46 & 30 & 2806 \\
\hline $4.5-5.0$ & $213.1-244.8$ & 1.74 & 61 & 36 & 63.4 & 6676 & 58.0 & 3872 & 2.2 & 147 & 3.15 & 210 & 2447 \\
\hline
\end{tabular}

Note: Wet-bulk density, porosity, and water content are from Site 532 chapter, this volume. Bulk-sediment MAR $=[(1-$ poros. $/ 100) \times 2.7] \times 100 \times($ sed. rate). MAR's for $\mathrm{CaCO}_{3}, \mathrm{C}_{\text {org }}$, and biogenic $\mathrm{SiO}_{2}=$ bulk-sediment $\mathrm{MAR} \times(\% / 100)$ of $\mathrm{CaCO}_{3}, \mathrm{C}_{\mathrm{org}}$, and biogenic $\mathrm{SiO}_{2}$, respectively. Percent $\mathrm{CaCO}_{3}$ and \% $\mathrm{C}_{\mathrm{org}}$ for each 0.5-m.y. interval are from Table 3. Percent biogenic $\mathrm{SiO}_{2}$ for each 0.5-m.y. interval were obtained from Dean and Parduhn, this volume. MAR "clay" is the difference between bulk-sed. MAR and the sum of MAR's for $\mathrm{CaCO}_{3}, \mathrm{C}_{\text {org }}$, and biogenic $\mathrm{SiO}_{2}$. 

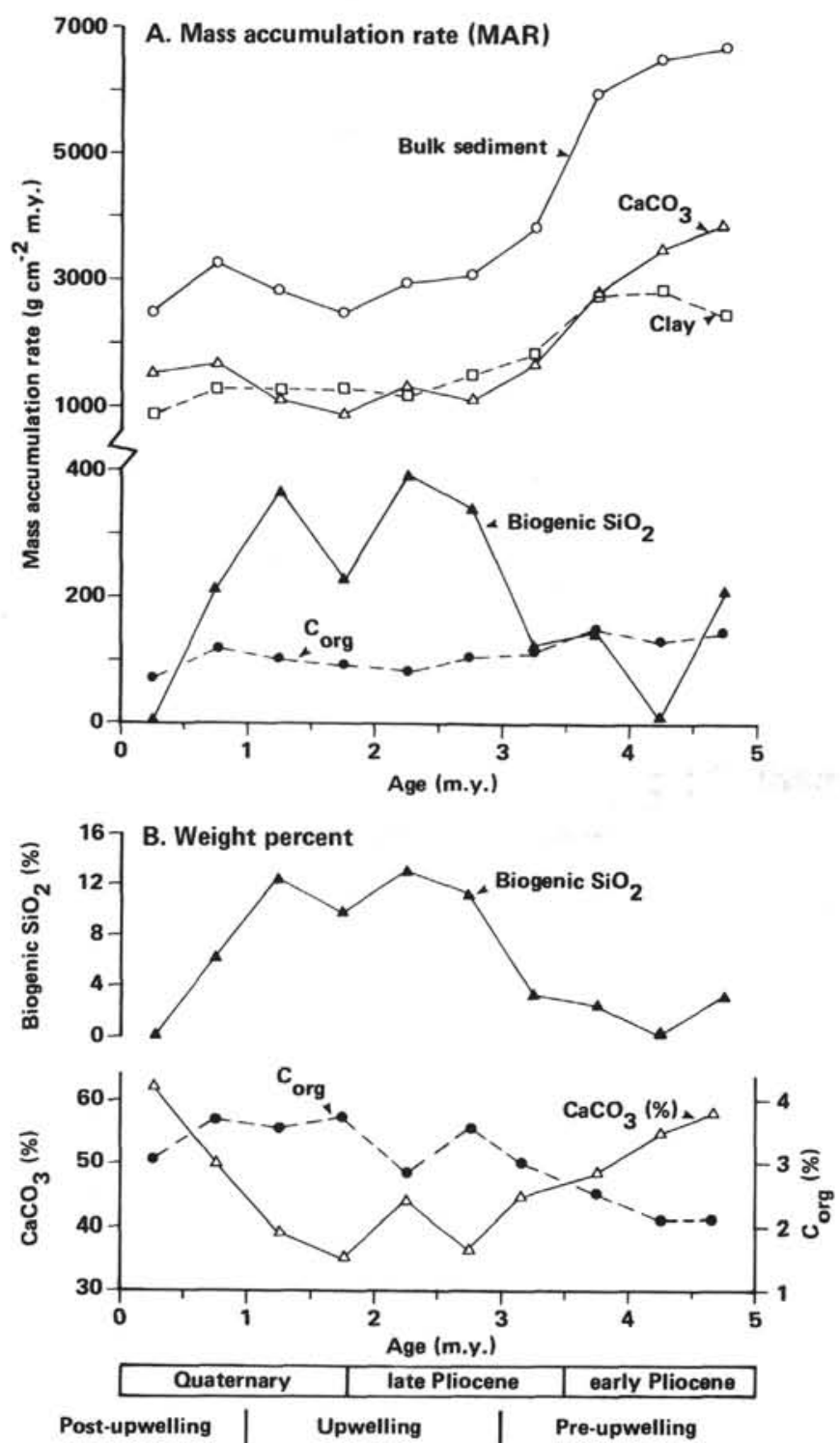

Figure 8. A. Plots of mass accumulation rates (MAR) of bulk sediment, $\mathrm{CaCO}_{3}$, organic carbon $\mathrm{C}_{\text {org }}$, biogenic silica, and clay vs. time for Hole 532. B. Plots of average weight percent $\mathrm{CaCO}_{3}$, organic carbon, and biogenic silica for each 0.5 m.y. interval for Hole 532. Data are from Table 5.

Neogene and Quaternary, as suggested by Gardner and Hays (1976), then the position of the core of the southeast Trades also would have fluctuated.

When upwelling migrated into the area of Site 532 between 3 and 2 m.y. ago, $\mathrm{CaCO}_{3}$ and clay MAR decreased by $60 \%$ and $47 \%$ respectively, and biogenic silica MAR increased by $144 \%$. However, because $\mathrm{CaCO}_{3}$ and clay had been accumulating in such large volumes during the pre-upwelling interval, the reduction in these components during the upwelling period was not offset by the increase of initially low biogenic silica, and the bulk MAR was reduced by $49 \%$ and the sedimentation rate dropped to $44 \mathrm{~m} / \mathrm{m}$.y. The cause of the reduction of $\mathrm{CaCO}_{3}$ and clay is problematical. One possibility for the reduction of $\mathrm{CaCO}_{3}$ is that the initiation of upwelling cooled the surface and near-surface waters to a

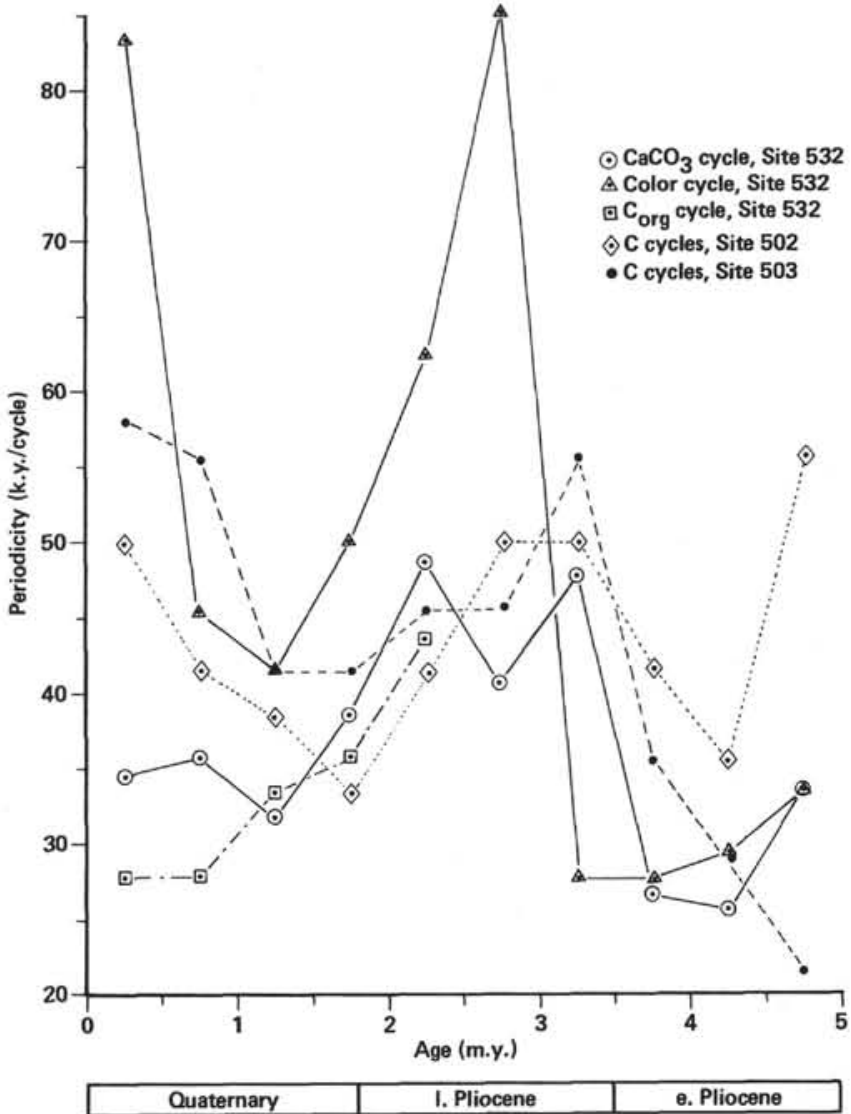

Figure 9. Plot of periodicities of 0.5 m.y. intervals of $\mathrm{CaCO}_{3}$ and organic carbon from Site 532, and from Site 502 in the eastern $\mathrm{Ca}$ ribbean and Site 503 in the eastern equatorial Pacific. Data for Site 532 are from Table 4; data for Sites 502 and 503 are from Gardner (1982).

degree that biogenic carbonate productivity was slowed. The increased flux of biogenic silica at this time would reflect the response of increased diatom productivity that took advantage of the cool, nutrient-rich, upwelling zone. But this does not account for the coeval reduction in the flux of clay. The timing of the beginning of upwelling, $2.5 \mathrm{~m} . y$. to $3.0 \mathrm{~m} . \mathrm{y}$. ago, is coincident with the inferred age for the initiation of glaciation in the Northern Hemisphere (Dalrymple, 1963; Curry, 1966; McDougall and Wensink, 1966; Kent et al., 1971; Berggren, 1972; Backman, 1979; Poore, 1978; 1981, among others). Northern Hemisphere glaciation had to have caused a drop in sea level as water was transferred from the oceans to land. Consequently, one would intuitively expect an increase, not a decrease, in the MAR of clay at this time. Perhaps the climate of southwest Africa became drier at this time, which would have reduced the flow of the Orange River and thus reduced sediment input to the continental margin. We have no data to substantiate this, and the reduction of clay at the beginning of upwelling in the area of Site 532 remains an unexplained enigma.

The upwelling interval is characterized by rather constant values of MAR for all components (Fig. 8), but the record of biogenic silica (Fig. 2) shows a series of large 


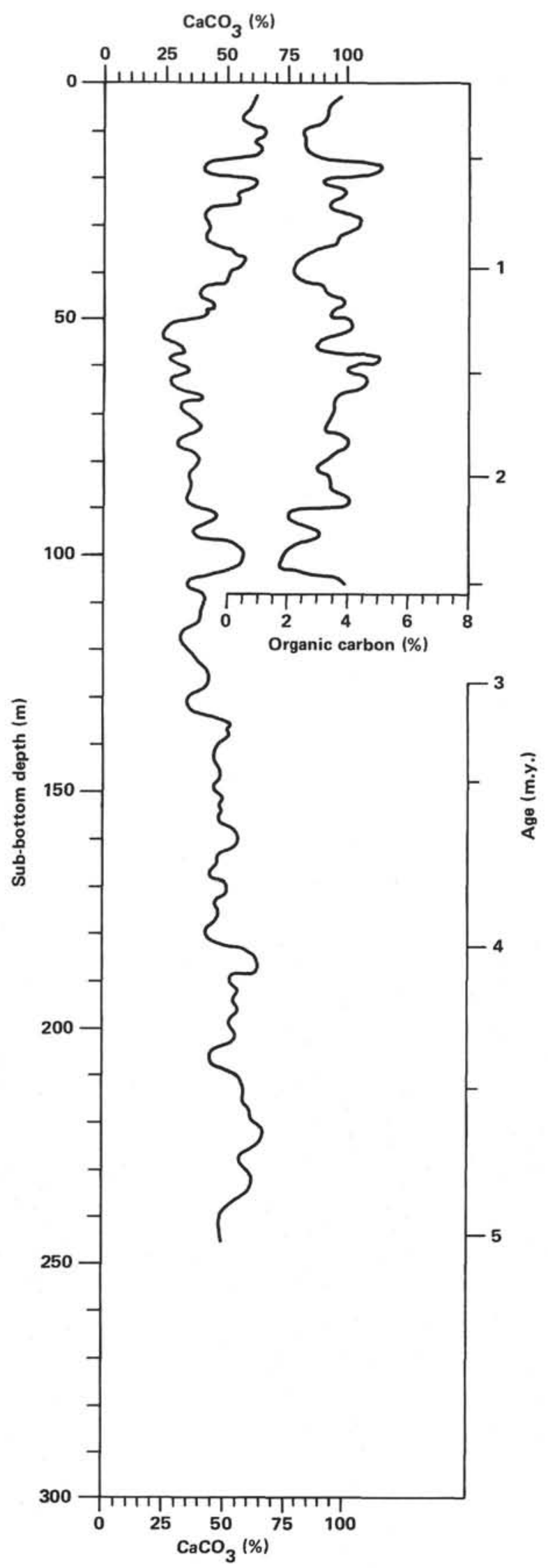

Figure 10. Smoothed curves of $\mathrm{CaCO}_{3}$ and organic-carbon percentages from Figure 2.

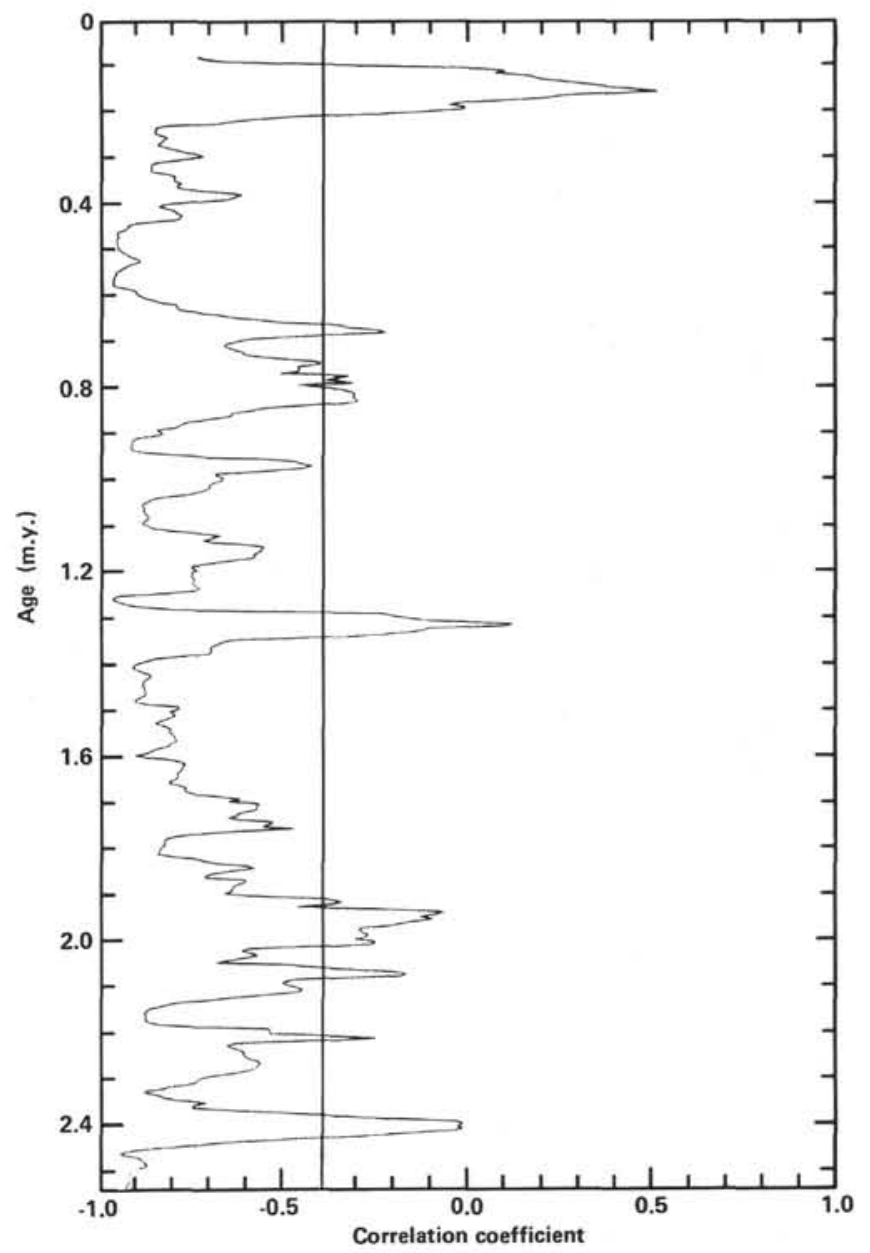

Figure 11. Moving correlation cefficients between $\mathrm{CaCO}_{3}$ and organic carbon using a 21-point window which represents about 100 k.y. The vertical line at a correlation coefficient of -0.43 represents the $95 \%$ confidence limit for correlation coefficients for $\mathrm{N}=21$.

fluctuations (cycles) that suggest upwelling intensity or position was not steady. An event occurred some time between $1.2 \mathrm{~m} . \mathrm{y}$. and $2.2 \mathrm{~m}$.y. ago which reduced total biogenic production but did not affect the flux of clay (Fig. 8). This event was probably a major fluctuation in either the strength or position of the center of upwelling; erosion can be discounted because the flux of clay was unaffected. Upwelling was reinstated in the area of Site 532 after the event, and continued until some time between $0.5 \mathrm{~m} . \mathrm{y}$. and $1.0 \mathrm{~m}$.y. ago. It should be emphasized that during the upwelling interval, the fluxes of $\mathrm{CaCO}_{3}$ and clay were still three to four times the flux of biogenic silica.

Mass accumulation rates for the post-upwelling interval (<1.0 m.y. ago) reflect a $99 \%$ reduction in biogenic silica relative to values during the upwelling period and a reduction of $35 \%$ in clay. The flux of $\mathrm{CaCO}_{3}$ during the post-upwelling period increased by $28 \%$ over the values during the upwelling interval, but the average sedimentation rate was only $34 \mathrm{~m} / \mathrm{m} . \mathrm{y}$.

The post-upwelling interval is complicated by a period of winnowing by bottom currents that affects sedi- 
ment from 0.5 m.y. old to the present. Apparently, currents as deep as $1300 \mathrm{~m}$ were intensified in the late Quaternary and have continued to winnow or inhibit deposition of fine-grained sediment since then. Winnowing has altered the composition of the sediment in this interval (Fig. 2) and consequently the MAR values in this interval may be misleading. The $99 \%$ reduction of biogenic silica and $35 \%$ reduction in clay MAR is certainly the result in part of the effect of erosion or nondeposition of fine-grained components. The apparent increase in $\mathrm{CaCO}_{3}$ MAR is a reflection of the lag of foraminifers left. It may be that the entire post-upwelling period is, in fact, not post-upwelling, but rather a continuation of the upwelling record that has been altered by erosion and/or nondeposition. However, the biogenic silica and diatom data (Fig. 2) suggest that upwelling in the area of Site 532 was losing intensity or migrating out of the area from about $1.2 \mathrm{~m} . \mathrm{y}$. to $0.5 \mathrm{~m}$.y. ago (before the pronounced effects of winnowing).

One of our goals was to find whether any component in the sediment could be used as a recorder of organic productivity. The Benguela Current upwelling system today produces massive upwelling of cold, nutrient-rich waters that result in high biological productivity (Brongersma-Sanders, 1957; Diester-Haass and Schrader, 1979; Brongersma-Sanders, et al., 1980; Siesser, 1980). In the open ocean at middle latitudes, diatoms take advantage of the upwelled supply of nutrients and their abundances in the underlying sediment overwhelm the other biogenic components (foraminifers, nannoplankton, and radiolarians). We do see increases in abundances of both diatoms and organic carbon in upper Pliocene and lower Quaternary sediment at Site 532 (Figs. 2 and 8), but not in the overwhelming proportions implied by the above citations. Either the plot of percent siliceous biogenic debris (mostly diatoms) or the plot of percent biogenic silica by XRF in Figure 2 can be used to obtain a semiquantitative measure of diatom productivity at Site 532, and presumably of upwelling intensity at Site 532.

Seisser (1980) suggested that Benguela Current upwelling began in early late Miocene (ca. 10 m.y. ago). Our data do not extend back that far, but percentages of diatoms and biogenic silica both show that diatom productivity was lower during the late Miocene and early Pliocene, reached a maximum in the late Pliocene and early Quaternary, and again diminished in the late Quaternary. The amount of organic carbon (Fig. 2) does not show a strong correlation with amount of clay, but does correlate with biogenic silica (Figs. 2 and 8 ) in that the average concentration of organic carbon is highest in the upper Pliocene to lower Quaternary. The overall correlation coefficient between organic carbon and biogenic silica, averaged over $0.5-\mathrm{m}$.y. intervals, is 0.60 .

Organic-carbon content has been correlated with sedimentation rate (Muller and Suess, 1979; Ibach, 1982), but, more interestingly, the data of Muller and Suess suggest that the combination of sedimentation rate and amount of organic carbon can be used to infer primary productivity, particularly for sedimentation rates great- er than about $40 \mathrm{~m} / \mathrm{m}$.y. When the data for Site 532 are plotted along with those of Muller and Suess on a sedimentation rate-organic-carbon diagram (Fig. 12), the Site 532 values are similar to those from the Canary Current upwelling system off northwest Africa. We can also project from Figure 12 that, as a very rough approximation, primary productivity at Site 532 ranged between 100 and $150 \mathrm{gC} / \mathrm{m}^{2} / \mathrm{y}$.

\section{CONCLUSIONS}

The entire section recovered at Site 532 on Walvis Ridge shows distinct cycles in amount of $\mathrm{CaCO}_{3}$ that generally correlate with cycles of sediment color. Average periodicities of the $\mathrm{CaCO}_{3}$ cycles range from 28.4 k.y./cycle in the lower Pliocene, to $45.6 \mathrm{k} . \mathrm{y} . /$ cycle in the upper Pliocene, to 35.1 k.y./cycle in the Quaternary, with an overall average of about $36 \mathrm{k} . \mathrm{y} . /$ cycle. Most minima of the carbonate cycles correspond to darker-colored beds, and most maxima of the carbonate cycles correspond to lighter-colored beds. These cycles in amount of carbonate persist even through periods of increased upwelling when there were large changes in the relative proportions of different sediment components.

Cycles in amount of organic carbon are not so distinct as those for $\mathrm{CaCO}_{3}$; maxima in organic-carbon concentration have an average periodicity of about 34 k.y./cycle for the last 2.5 m.y. All dark-colored beds correspond to organic-carbon maxima, but many maxima do not appear to correspond to a dark bed. The profiles of both $\mathrm{CaCO}_{3}$ and organic carbon show longerperiod cycles with average periodicities of about 150 k.y./cycle. In general, concentrations of organic carbon and $\mathrm{CaCO}_{3}$ are inversely related, both on a short-term
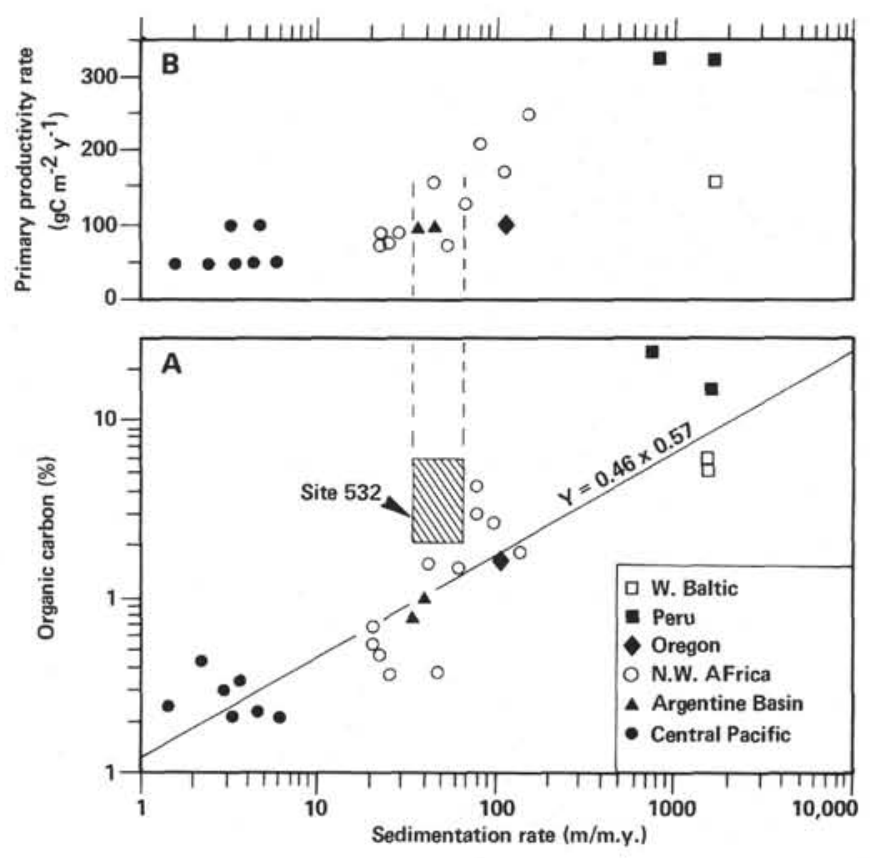

Figure 12. Relations among sedimentation rate, organic-carbon content, and primary productivity for recent marine sediments (modified from Müller and Suess, 1979). 
basis within individual cycles and on a long-term basis over the entire recovered section.

The carbonate cycles are not the result of carbonate dissolution, but rather some complex combination of influx of terrigenous clastics and carbonate productivity. Because of the similarity of the periodicities of the Site 532 cycles to those of carbonate cycles in the northeast Atlantic, Caribbean, and eastern equatorial Pacific oceans, these cycles probably are responses to global events. Fluctuations in global sea level is one possible forcing mechanism for the $\mathrm{CaCO}_{3}$ cyclicity at Site 532 because of the correlation of the carbonate cycles to clay input.

The carbonate record shows no apparent response to the Benguela-Current upwelling history in this region. Organic carbon does show a gross correlation to inferred upwelling in that there is a positive correlation between organic carbon and biogenic silica. The best indicators of upwelling intensity are semiquantitative estimates of diatom abundances from smear slides, calculated concentrations of biogenic $\mathrm{SiO}_{2}$, and the ratio $\mathrm{SiO}_{2}: \mathrm{Al}_{2} \mathrm{O}_{3}$. These indicators all suggest that upwelling at Site 532 was minor until about $2.5 \mathrm{~m}$.y. ago (late Pliocene). Diatom abundances, biogenic $\mathrm{SiO}_{2}$, and organic carbon increase in the upper Pliocene section, and the average values remain high, but with fluctuations until mid-Quaternary. This period, $2.5 \mathrm{~m} . \mathrm{y}$. to $0.5 \mathrm{~m} . \mathrm{y}$., appears to be a time of relatively intense upwelling at Site 532. Upwelling may have occurred before $2.5 \mathrm{~m} . \mathrm{y}$. along other segments of the southwest African continental margin, as it does today. The center of upwelling may have migrated in response to the location of the main core of the southeast Trade Winds.

The relationships between sedimentation rate, productivity, and organic-carbon content described by Muller and Suess (1979) indicate that during the period of maximum upwelling, the primary productivity at Site 532 ranged between 100 and $150 \mathrm{gC} / \mathrm{m}^{2} / \mathrm{yr}$., a value similar to that occurring today of northwest Africa in the Canary-Current upwelling system.

The top $20 \mathrm{~m}$ of the recovered section at Site 532 is a foraminiferal-rich facies that suggests strong currents have occurred at this depth $(1331 \mathrm{~m})$ some time during the past $500 \mathrm{k} . y$. This increased circulation was capable of transporting and possibly eroding nannofossils and diatoms, but not foraminifers.

\section{ACKNOWLEDGMENTS}

We thank the shipboard scientists on DSDP Leg 90 for all the stimulating discussions during the cruise. In addition, we appreciate the thoughtful reviews by M. A. Arthur and R. Z. Poore.

\section{REFERENCES}

Anderson, R. Y., 1982. A long geoclimatic record from the Permian. J. Geophys. Res., 87:7285-7294.

Arthur, M. A., 1979. North Atlantic Cretaceous black shales: The record at Site 398 and a brief comparison with other occurrences. In Sibuet J.-C., Ryan, W. B. F., et al., Init. Repts. DSDP, 47, Pt. 2: Washington (U.S. Govt. Printing Office), 719-751.

Arthur, M. A., and Fischer, A. G., 1977. Upper Cretaceous-Paleocene magnetic stratigraphy at Gubbio, Italy: I. Lithostratigraphy and sedimentology. Geol. Soc. Am. Bull., 88:367-389.
Backman, J., 1979. Pliocene biostratigraphy of DSDP Sites 111 and 116 from the North Atlantic Ocean and the age of northern Hemisphere glaciation. Stockholm Contrib. Geol., Acta Universitatis Stockholmiensis, 32:115-137.

Berggren, W. A., 1972. Late Pliocene-Pleistocene glaciation. In Laughton, A. S., Berggren, W. A., et al., Init. Repts. DSDP, 12: Washington (U.S. Govt. Printing Office), 953-963.

Bolli, H. M., Ryan, W. B. F., et al., 1978. Init. Repts. DSDP, 40: Washington (U.S. Govt. Printing Office).

Boström, K., Joensuu, O., Valdes, S., and Riera, M., 1972. Geochemical history of South Atlantic Ocean sediments since the late Cretaceous. Mar. Geol., 12:85-122.

Broecker, W. S., and van Donk, J., 1970. Insolation changes, ice volumes and the $\mathrm{O}^{18}$ record in deep-sea cores. Rev. Geophys. Space Phys., 8:169-198.

Brongersma-Sanders, M. 1957. Mass mortality in the sea. In Hedgepeth, J. W. (Ed.), Treatise on Marine Ecology and Paleoecology (Vol. 2). Mem. Geol. Soc. Am., 67:941-1010.

Brongersma-Sanders, M., Stephan, K. M., Kwee, T. G., and de Bruin, M., 1980. Distribution of minor elements in cores from the Southwest Africa shelf with notes on plankton and fish mortality. Mar. Geol., 37:91-132.

Calvert, S. E., and Price, N. B., 1970. Minor metal contents of recent organic-rich sediments off Southwest Africa. Nature, 227:593-595.

Clifton, E., 1980. Progradational sequences in Miocene shoreline deposits, southeastern Caliente Range, California. J. Sediment. Petrol., 51:166-184.

Curry, R. R., 1966. Glaciation about 3,000,000 years ago in the Sierra Nevada. Science, 154:770-771.

Dalrymple, G. B., 1963. Potassium-argon dates of some Cenozoic volcanic rocks of the Sierra Nevada. Geol. Soc. Am. Bull. 74: 379-390.

Dean, W. E., and Anderson, R. Y., 1974. Application of some correlation coefficient techniques to time-series analysis. Math. Geol., 6:363-372.

Dean, W. E., Gardner, J. V., and Ćepek, P., 1981. Tertiary carbonate-dissolution cycles on the Sierra Leone Rise, eastern equatorial Atlantic Ocean. Mar. Geol., 39:81-101.

Dean, W. E., Gardner, J. V., Jansa, L. F., Čepek, P., and Seibold, E., 1978. Cyclic sedimentation along the continental marine of northwest Africa. In Lancelot, Y., Seibold, E., et al., Init. Repts. DSDP, 41: Washington (U.S. Govt. Printing Office), 965-989.

deVries, T. J., and Schrader, H., 1981. Variation of upwelling/oceanic conditions during the latest Pleistocene through Holocene off the central Peruvian Coast: A diatom record. Mar. Micropaleont., 6:157-167.

Diester-Haass, L., and Schrader, H. J., 1979. Neogene coastal upwelling history off northwest and southwest Africa. Mar. Geol., 29: 39-53.

Ellis, H., 1981. Calcareous nannoplankton biostratigraphy-DSDP Leg 60. In Hussong, D. M., Uyeda, S., et al., Init. Repts. DSDP, 60: Washington (U.S. Govt. Printing Office), 507-535.

Fischer, A. G., 1964. The Lofer cyclothems of the Alpine Triassic. In Merriam, D. F. (Ed.), Symposium on Cyclic Sedimentation. Bull.-Kans., State Geol. Surv., 169, 1:107-149.

Gardner, J. V., 1982. High-resolution carbonate and organic-carbon stratigraphies for the late Neogene and Quaternary from the western Caribbean and eastern equatorial Pacific. In Prell, W. L., Gardner, J. V., et al., Init. Repts. DSDP, 68: Washington (U.S. Govt. Printing Office)., 347-364.

Gardner, J. V., and Hays, J. D., 1976. Responses of sea-surface temperature and circulation to global climatic change during the past 200,000 years in the eastern equatorial Atlantic Ocean. In Cline, R. M., and Hays, J. D. (Eds.), Investigations of Late Quaternary Paleoceanography and Paleoclimatology. Mem. Geol. Soc. Am., 145:221-246.

Hays, J. D., Imbrie, J., and Shackleton, N. J., 1976. Variations in the earth's orbit: Pacemaker of the ice ages. Science, 194:1121-1132.

Hülsemann, J., 1966. On the routine analysis of carbonates in unconsolidated sediments. J. Sediment. Petrol., 36:622-625.

Ibach, L. E. J., 1982. Relationship between sedimentation rate and total organic-carbon content in ancient marine sediments. Am. Assoc. Petrol. Geol. Bull., 66:170-188. 
Kent, D., Opdyke, N. D., and Ewing, M., 1971. Climate change in the North Pacific using ice-rafted detritus as a climatic indicator. Geol. Soc. Am. Bull., 82:2741-2759.

McCave, I. N., 1979. Depositional features of organic-rich black and green mudstones at DSDP Sites 386 and 387, western North Atlantic. In Tucholke, B. E. and Vogt, P. R., et al., Init. Repts. $D S D P, 43$ : Washington (U.S. Govt. Printing Office), 411-416.

McDougall, I. and Wensink, H., 1966. Paleomagnetism and geochronology of the Pliocene-Pleistocene lava in Iceland. Earth Planet. Sci. Lett., 1:232-236.

Martini, E., 1971. Standard Tertiary and Quaternary calcareous nannoplankton zonation. Second Plankt. Conf.: Roma (Technoscienzia), pp. 739-785.

Matthews, R. K., 1982. The oxygen isotope record of ice volume history: 100 million years of glacio-eustatic sea-level fluctuations. Mem. Am. Assoc. Petrol. Geol., 66:2.

Matthews, R. K., and Poore, R. Z., 1980. Tertiary ${ }^{18} \mathrm{O}$ record and glacio-eustatic sea-level fluctuations. Geology, 8:501-504.

Müller, G., and Gastner, M., 1971. The "karbonate bomb", a simple device for the determination of carbonate content in sediment, soils, and other materials. N. Jahrb. Mineral. Mh., 10:466-469.

Müller, P. S., and Suess, E., 1979. Productivity, sedimentation rate, and sedimentary organic carbon content in the oceans. Deep-Sea Res., 26:1347-1362.

Poore, R. Z., 1978. Oligocene through Quaternary planktonic foraminiferal biostratigraphy of the North Atlantic: DSDP Leg 49. In Luyendyk, B. P., Cann, J. R., et al., Init. Repts. DSDP, 49: Washington (U.S. Govt. Printing Office), 447-518.
1981. Temporal and spatial distribution of ice-rafted mineral grains in Pliocene sediments of the North Atlantic: Implications for Late Cenozoic climatic history. SEPM Spec. Publ., 32: 505-515.

Prell, W. L., 1982. Oxygen and carbon isotope stratigraphy for the Quaternary of Hole 502B: Evidence for two modes of isotopic variability. In Prell, W. L., Gardner, J. V., et al., Init Repts. $D S D P, 68$ : Washington (U.S. Govt. Printing Office).

Rea, D. K., and Janecek, T. R., 1981. Mass-accumulation rates of the non-authigenic inorganic crystalline (eolian) component of deep-sea sediments from the western Mid-Pacific Mountains, Deep Sea Drilling Project Site 463. In Thiede, J., Vallier, T. L., et al., Init. Repts. DSDP, 62: Washington (U.S. Govt. Printing Office), 653-659.

Schuette, G., and Schrader, H., 1981. Diatom taphocoenoses in the coastal upwelling area off southwest Africa. Mar. Micropaleont. 6:133-155.

Seisser, W. G., 1980. Late Miocene origin of the Benguela Upwelling System off northern Namibia. Science, 208:283-285.

Shackleton, N. J., 1967. Oxygen isotope analyses and Pleistocene temperatures reassessed. Nature, 215:15-17.

Tissot, B., Durand, B., Espitalié, J., and Combaz, A., 1974. Influence of nature and diagenesis of organic matter information of petroleum. Amer. Assoc. Petroleum Geol. Bull., 58:499-506.

van Andel, Tj. H., Heath, G. R., and Moore, T. C., Jr., 1975. Cenozoic history and paleoceanography of the central equatorial $\mathrm{Pa}$ cific Ocean. Men. Geol. Soc. Am., 143. 